\title{
Semi-analytical model of mixed electroosmotic/pressure driven two immiscible fluids with curved interface
}

\author{
School of Mechanical and Aerospace Engineering, Nanyang Technological University \\ 50 Nanyang, Avenue, Singapore 639798, \\ *Corresponding author. E-mail: $\underline{\text { mtnwong@ntu.edu.sg }}$
}

\begin{abstract}
This study is motivated by the need to develop a semi analytical model for predicting the two-fluid flow with a curved interface in a rectangular stratified microchannel under the combined effect of pressure driven and electroosmotic effect. With the non-slip boundary conditions at the wall and the matching condition at the curved interface, the fully developed Navier-Stokes equation and Poisson-Boltzmann equation are solved using separate variable method. Part of parameters in the distributions of velocity and electric potential are calculated using the least-squares numerical method. Details of the analytical treatment of the two-fluid flow with curved interface are presented and it is shown that the analysis can be employed for concave, convex and plane interface. The validity of the two-fluid model with curved interface is evaluated by comparing its prediction with available numerical data and with the results of exact analytical solutions for laminar flows with plane interface; comparison of the electric potential distribution and velocity distributions show excellent agreement with data in the literature. Finally, the effects of interface shape to the electric potential distribution, electroosmotic velocity distribution, and flow rates are discussed; the results show that the interface shape influences the two-fluid flow in microchannel significantly.
\end{abstract}

Keywords Curved interface; two - fluid stratified flow; electroosmotic flow; microchannel 


\section{Introduction}

Microfluidic and Lab-on-a-chip devices have focused increasing interest over the last decade due to the advantages of increased efficiency, throughput, portability, and reduced analysis time, reagent consumption, and cost. Comparing with a number of techniques (e.g., pressure, centrifuge, and thermal gradient driven flow), electroosmosis has been used to induce electroosmotic flow because electroosmosis does not involve any moving mechanical parts and generates the plug-like velocity profile [1-3]. Two-fluid flow in microchannel is used as micro-pump [4-6], switching technique [7-9], mixing [10-12] and so on. Models of stratified flow are needed for predicting the flow characteristics, such as pressure drop and in-situ liquid fraction, and are often used as a starting point in modeling flow patterns transitions. The interface shape is one of crucial factor on flow pressure drop and in-situ liquid fraction.

The common assumption is that the interface separating the phases is planar $[8,13-$ 15]. Under this assumption, Lee et al. [16] applied flow focusing to develop various valveless micro flow switches; Gao et al. $[14,17]$ derived the analytical solution of the velocity profile and flowrate of two-liquid flow in microchannels which was driven both by electroosmotic force and pressure gradient; Wang et al [8] controlled the interface using the electroosmotic effect. In our previous work $[15,18]$, a three-fluid flow in microchannels under the combined effect of pressure driven and electroosmotic effect were presented, planar interfaces are assumed.

The effect of interface curvature has been studied in macro or mini channels by lots of researchers. Bentwich [19] investigated the two-phase flow with interface defined by a circular arc or an eccentric circle using analytical method; the interfacial tension, capillary force and gravity are included in this model. Ong et al [20] solved the characteristics of straight liquid-liquid flow with curved interface using numerical method. Brauner et al [21] analytically solved the two-phase flow characteristics in stratified two-phase flow for curved interface with the interface curvature as a prescribed parameter. Brauner et al [22-23] provided a predictive tool for determining the characteristic interface curvature in two phase systems; the steady interfacial curvature is shown to correspond to the interface configuration for which the total system energy is at its minimum; the papers assumed that the interface is an approximate circular arc. 
Gorelik et al [24] obtained the exact analytical solution of the interface shape and capillary pressure between the two immiscible fluids. By solving the Young-Laplace equation, $\mathrm{Ng}$ et al [25] presented different exact interface shapes for various Bond numbers, contact angles and liquid fractions; the results show that for small Bond numbers, surface tension is dominant and the interface approaches a circular arc shape. Using the boundary element method $\mathrm{Ng}$ et al [26] analyzed the volumetric flow rates, the wall shear stresses and the velocity profiles of the interface using actual interface shape.

The review shows that most previous works focused on macro or mini channels. In comparison with macro/mini channels, the effect of interface curvature in microchannel is very significant but little work has been reported in this area.

For flow in microchannels, the gravity effect is low [27] while the surface tension is strong which causing the Bond number approaches to zero. The results of [22-25] showed that the interface is a circular arc with constant curvature in microchannels.

The aim of the work is to present a precise calculation method for two-fluid laminarlaminar stratified flow under the combined effect of pressure driven and electroosmotic effect where the interfaces shape is a circular arc with a constant curvature. Fig. 1 shows the general configuration of the fluids in microchannels with curved interface. The fluid with high electrical mobility is labeled fluid 1 , while the fluid with low electrical mobility is labeled fluid 2. The flows are assumed to be at a steady state, fully developed, and therefore unidirectional.

\section{Mathematical model}

\subsection{The description of the model}

In microchannel, the effect of gravity is ignored and the location of the interface is determined by the fluids/wall wettability angle and the fluids liquid fraction only. It is assumed that the curvature along the interface is constant in microchannels [22-25].

In order to analyze the system shown in Fig.1, a Cartesian coordinate system $(x, y$, $z$ ) is used where the origin point, $O$, is set to be at the centre of the interface curvature. Curved interface with constant curvature is assumed. $h_{1}, h_{2}$, and $h_{\mathrm{s}}$ are defined in Fig. 2; the relationship between them is $h_{1}+h_{2}+h_{\mathrm{s}}=h$ where $h_{\mathrm{s}}$ is the height of the curved 
interface measured from the origin and $h$ is the height of the microchannel. The width of the channel is denoted by $2 w$. The contact angle $\theta_{c}$ is the angle at which the fluid 1 meets the wall. As a result of surface charges, electric double layers (EDLs) form next to the liquid-liquid interface and the channel walls that are in contact with the high electrical mobility fluid. For a more general situation, the walls of the microchannel may be made of different materials, so that the zeta potentials at the bottom wall is denoted as $\xi_{1}$, and at the side walls as $\xi_{2}, \xi_{4}$, respectively. The zeta potentials at the curved interface is $\xi_{3}$. The flows are along the $z$ direction.

The two fluids are driven by the combined pressure and electroosmotic body forces. When the two-fluid flow is fully developed, the velocities of the two liquids, $u_{1}$ and $u_{2}$ at position $(x, y)$ along the channel are independent of $z$. The subscripts of 1 and 2 denote the fluid 1 and fluid 2, respectively.

The length scale and velocity scale of the flow are taken as $L_{\text {ref }}$ and $U_{\text {ref }}$, respectively. The independent variable $x$ and $y$ and dependent variables $u, p, \psi$ and $\rho_{q}$ are expressed in terms of the corresponding dimensionless quantities (shown with an over bar ) by

$$
\left\{\begin{array}{l}
x=L_{r e f} \bar{x} \\
y=L_{r e f} \bar{y} \\
u=U_{r e f} \bar{u} \\
\psi=k_{b} T \bar{\psi} /\left(z_{0} e\right) \\
p=\rho_{1} U_{r e f}^{2} \bar{p} \\
\rho_{q}=2 n_{0} z_{0} e \bar{\rho}_{q}
\end{array}\right.
$$

where $\rho$ is the liquid density, $k_{b}$ is Boltzmann constant, $T$ is the absolute temperature, $z_{0}$ is the valence of the ions, $e$ is elementary charge, and $n_{0}$ is the reference value of the ion concentration.

The interface is concave with contact $\theta_{c}<90^{\circ}$ (Fig. 2(a)) and convex with contact $\theta_{c}>90^{\circ}$ (Fig. 2(c)). Fig. 2(b) corresponds to the plane interface with $\theta_{c}=90^{\circ}$. There are two restrictive conditions as shown in Fig. 3: one is that the interface contact with the bottom wall (Fig. 3(a)) and the other is that the interface contact with the top wall (Fig. 3(b)). In our study, the interface is located between Fig. 3(a) and Fig. 3(b). 
In order to derive expediently, the following derivation of analytical model is completed in polar coordinate. Two parameters are defined as follows

$$
\bar{r}=\sqrt{\bar{x}^{2}+\bar{y}^{2}}
$$

and

$$
\theta= \begin{cases}0 & (\bar{x} \geq 0, \bar{y}=0) \\ \arctan \frac{\bar{y}}{\bar{x}} & (\bar{x}>0, \bar{y}>0) \\ \frac{\pi}{2} & (\bar{x}=0, \bar{y}>0) \\ \pi-\arctan \frac{\bar{y}}{|\bar{x}|} & (\bar{x}<0, \bar{y}>0) \\ \pi \arctan \mid \frac{\bar{y} \mid}{\bar{x} \mid} & (\bar{x}<0, \bar{y}=0) \\ \frac{3 \pi}{2} & (\bar{x}=0, \bar{y}<0) \\ 2 \pi-\arctan \frac{|\bar{y}|}{\bar{x}} & (\bar{x}<0, \bar{y}<0)\end{cases}
$$

where $r$ and $\theta$ are the radius and angle in the polar coordinate. In the derivation, $\mathrm{r}$ and $\theta$ are used to replace $x$ and $y, \bar{x}$ and $\bar{y}$ are the dimensionless position of arbitrary point in $\mathrm{x}$-axis and $\mathrm{y}$-axis, respectively.

\subsection{Electric potential distributions}

The electric potential in the high electrical mobility fluid 1 is first considered. Assuming that the electric charge density is not affected by the external electric field due to the thin EDLs and the small fluid velocity, the charge convection can be ignored and the electric field and fluid flow equations are decoupled [28-29]. Based on the assumption of local thermodynamic equilibrium, for a small zeta potential, the electric potentials $\bar{\psi}$ due to the charged wall are described by the linear Poisson-Boltzmann equation which can be written in terms of dimensionless variables as

$$
\nabla^{2} \bar{\psi}=K^{2} \bar{\psi}
$$


where $K=L_{r e f} \kappa$ is the ratio of the length scale $L_{r e f}$ to the characteristic double-layer thickness $1 / \kappa$. For this case, the reference length is chosen as $L_{r e f}=w$. Here, $\kappa$ is the Debye-Hückel parameter,

$$
\frac{1}{\kappa}=\left(\frac{\varepsilon k_{b} T}{2 z_{0}^{2} e^{2} n_{0}}\right)^{\frac{1}{2}}
$$

In cylindrical coordinates, Eq. (4) is specified as

$$
\frac{1}{r} \frac{\partial}{\partial r}\left(r \frac{\partial \bar{\psi}}{\partial r}\right)+\frac{1}{r^{2}} \frac{\partial^{2} \bar{\psi}}{\partial \theta^{2}}=K^{2} \bar{\psi}
$$

Eq. (6) is the modified Bessel's equation. Using the Separated Variable Method in polar coordinate, the solution of Eq. (6) can be described as

$$
\bar{\psi}(r, \theta)=\sum_{\lambda=0}^{\infty} I_{\lambda}(K r)\left[A_{1 \lambda} \cos (\lambda \theta)+A_{2 \lambda} \sin (\lambda \theta)\right]
$$

The boundary condition of zeta potential can be defined as

$$
\bar{\psi}\left(r_{i}, \theta_{i}\right)=Z_{i}
$$

where the subscribe of $i$ is the serial number of the boundary condition. Using the description of Eq. (7), Eq. (8) transforms into

$$
\sum_{\lambda=0}^{J} I_{\lambda}\left(K r_{i}\right)\left[A_{1 \lambda} \cos \left(\lambda \theta_{i}\right)+A_{2 \lambda} \sin \left(\lambda \theta_{i}\right)\right]=\bar{\psi}\left(r_{i}, \theta_{i}\right)
$$

where $I_{\lambda}(K r)$ is the modified Bessel functions of order $\lambda$ of the first kind. The real coefficients of $A_{1 \lambda}$ and $A_{2 \lambda}$ can be numerically determined from the Dirichlet boundary conditions of the specified value of zeta potential on the channel walls and interface, which can be calculated using the least square method in Appendix A and Appendix B.

\subsection{Velocity driven by pressure gradient $\left(\bar{u}_{1}^{p}\right.$ and $\left.\bar{u}_{2}^{p}\right)$}

Due to linearity, the velocity of the high electrical mobility fluid 1 and of the low electrical mobility fluid 2 can be decomposed into two parts:

$$
\bar{u}=\bar{u}^{p}+\bar{u}^{E}
$$

where $\bar{u}^{E}$ corresponds to the velocity driven by electroosmotic force, and $\bar{u}^{p}$ is the velocity driven by pressure gradient. In this part, the velocity driven by pressure gradient is considered independently. 
The steady state Navier-Stokes equation with pressure driven can be described as

$$
\nabla^{2} \bar{u}_{1}^{p}=\operatorname{Re} \frac{d \bar{p}}{d \bar{z}} \quad \text { (fluid 1) }
$$

and

$$
\nabla^{2} \bar{u}_{2}^{p}=\frac{\operatorname{Re}}{\beta} \frac{d \bar{p}}{d \bar{z}} \quad \text { (fluid 2) }
$$

where $\beta$ is the dynamic viscosity ratio $\beta=\mu_{2} / \mu_{1}, \operatorname{Re}$ is the Reynolds number, $\operatorname{Re}=\frac{\rho_{r e f} U_{r e f} L_{r e f}}{\mu_{r e f}}, \frac{d \bar{p}}{d \bar{z}}$ is pressure gradient along flow direction. In polar coordinates, Eq. (11) is specified as

$$
\frac{1}{r} \frac{\partial}{\partial r}\left(r \frac{\partial \bar{u}_{1}^{p}}{\partial r}\right)+\frac{1}{r^{2}} \frac{\partial^{2} \bar{u}_{1}^{p}}{\partial \theta^{2}}=\operatorname{Re} \frac{d \bar{p}}{d \bar{z}}
$$

In order to convert Eq. (13) to a Laplace equation, the following transformation is used

$$
\bar{u}_{1}^{p}=\operatorname{Re} \frac{d \bar{p}}{d \bar{z}}\left(\frac{r^{2}}{4}-\bar{u}_{1}^{p^{*}}\right)
$$

Substituting Eq. (14) into Eq. (13), it transforms into

$$
\frac{1}{r} \frac{\partial}{\partial r}\left(r \cdot \frac{\partial \bar{u}_{1}^{p^{*}}}{\partial r}\right)+\frac{1}{r^{2}} \frac{\partial^{2} \bar{u}_{1}^{p^{*}}}{\partial \theta^{2}}=0
$$

Eq. (15) is the modified Bessel's equation. Using the Separated Variable Method in polar coordinate, the solution of Eq. (15) can be described as

$$
\bar{u}_{1}^{p^{*}}=a_{10}^{p}+\sum_{j=1}^{J} r^{j}\left(a_{1 j}^{p} \cos (j \theta)+b_{1 j}^{p} \sin (j \theta)\right)
$$

According Eq. (14)

$$
\bar{u}_{1}^{p}=\operatorname{Re} \frac{d p}{d z}\left(\frac{r^{2}}{4}-a_{10}^{p}-\sum_{j=1}^{J} r^{j}\left(a_{1 j}^{p} \cos (j \theta)+b_{1 j}^{p} \sin (j \theta)\right)\right)
$$

The non-slip boundary conditions can be used as

$$
a_{10}^{p}+\sum_{j=1}^{J} r^{j}\left(a_{1 j}^{p} \cos (j \theta)+b_{1 j}^{p} \sin (j \theta)\right)=\frac{r_{i}^{2}}{4} \quad \text { (for fluid 1) }
$$

The parameters of $a_{10}, a_{1 \mathrm{j}}$ and $b_{1 \mathrm{j}}$ are constants for specified boundary conditions.

Using the following method, the hydrodynamic velocity of fluid 2 is 


$$
\bar{u}_{2}^{p}=\frac{\operatorname{Re}}{\beta} \frac{d p}{d z}\left(\frac{r^{2}}{4}-a_{20}^{p}-\sum_{j=1}^{J} r^{j}\left(a_{2 j}^{p} \cos (j \theta)+b_{2 j}^{p} \sin (j \theta)\right)\right)
$$

The non-slip boundary conditions can be expressed as

$$
a_{20}^{p}+\sum_{j=1}^{J} r^{j}\left(a_{2 j}^{p} \cos (j \theta)+b_{2 j}^{p} \sin (j \theta)\right)=\frac{r_{i}^{2}}{4} \quad \text { (for fluid 2) }
$$

The matching conditions at the interface can be described as

$$
\left\{\begin{array}{l}
\bar{u}_{1}^{p}\left(r_{i}, \theta_{i}\right)=\bar{u}_{2}^{p}\left(r_{i}, \theta_{i}\right) \\
\frac{\partial \bar{u}_{1}^{p}\left(r_{i}, \theta_{i}\right)}{\partial n}=\beta \frac{\partial \bar{u}_{2}^{p}\left(r_{i}, \theta_{i}\right)}{\partial n} \quad \text { (at the interface) }
\end{array}\right.
$$

According Eqs. (18), (20) and the non-slip boundary conditions can be described using the following equations

$$
\left\{\begin{array}{l}
a_{10}^{p}+0 \cdot a_{20}^{p}+\sum_{j=1}^{J} r_{i}^{j}\left(a_{1 j}^{p} \cos (j \theta)+b_{1 j}^{p} \sin (j \theta)+a_{2 j} \cdot 0+b_{2 j} \cdot 0\right)=\frac{r_{i}^{2}}{4} \quad \text { (fluid 1) } \\
0 \cdot a_{10}^{p}+a_{20}^{p}+\sum_{j=1}^{J} r_{i}^{j}\left(a_{1 j} \cdot 0+b_{1 j} \cdot 0+a_{2 j}^{p} \cos (j \theta)+b_{2 j}^{p} \sin (j \theta)\right)=\frac{r_{i}^{2}}{4} \quad \text { (fluid 2) }
\end{array}\right.
$$

The matching conditions of Eq. (21) can be described using the following equation

$$
\beta \cdot a_{10}^{p}-a_{20}^{p}+\sum_{j=1}^{J}-\left(\beta \cdot r_{i}^{j} \cdot \cos \left(j \theta_{i}\right)+C_{1}^{p}(j)\right) \cdot a_{2 j}^{p}-\left(r_{i}^{j} \cdot \sin \left(j \theta_{i}\right)+S_{1}^{p}(j)\right) b_{2 j}^{p}=(\beta-1) \cdot \frac{r_{i}^{2}}{4}
$$

where $C_{1}(j)$ and $S_{1}(j)$ can be described as

$$
\begin{aligned}
& C_{1}^{p}(j)=r_{i}^{j-1} \cdot \cos \left(j \theta_{i}\right) \cdot j \cdot \cos \theta_{i}+r_{i}^{(j-2)} \cdot \sin \left(j \theta_{i}\right) \cdot j \cdot \sin \theta_{i} \\
& S_{1}^{p}(j)=r_{i}^{j-1} \cdot \sin \left(j \theta_{i}\right) \cdot j \cdot \cos \theta_{i}-r_{i}^{(j-2)} \cdot \cos \left(j \theta_{i}\right) \cdot j \cdot \sin \theta_{i}
\end{aligned}
$$

The real coefficients of $a_{10}^{p}, a_{20}^{p}, a_{1 j}^{p}, b_{1 j}^{p}, a_{2 j}^{p}$, and $b_{2 j}^{p}$ in Eq. (22) can be calculated using the least square method in Appendix A and Appendix C, and then the velocity profile due to pressure gradient can be described using Eqs. (17) and (19).

\subsection{Velocity driven by electroosmotic force $\left(\bar{u}_{1}^{E}\right.$ and $\left.\bar{u}_{2}^{E}\right)$}

The steady state Navier-Stokes equation under external electric field can be described as

$$
\mu \nabla^{2} u_{1}^{E}=-\rho_{q} E
$$


where $\rho_{q}$ is the bulk density of charge, $E$ is the external electric field. The Poisson's equation is

$$
\nabla^{2} \psi=-\frac{\rho_{q}}{\varepsilon_{0} \varepsilon_{r}}
$$

Combining Eqs. (26) and (27), the Navier-Stokes equation transforms into

$$
\mu \nabla^{2} u_{1}^{E}=\varepsilon_{0} \varepsilon_{r} \cdot \nabla^{2} \psi \cdot E
$$

The dimensionless form of Eq. (28) is

$$
\frac{1}{\operatorname{Re}} \bar{\mu} \nabla^{2} \bar{u}_{1}^{E}=\bar{E} \cdot \nabla^{2} \bar{\psi}
$$

where $\bar{E}=\frac{k_{b} T \varepsilon_{0} \varepsilon_{r} E}{L_{r e f} U_{r e f}^{2} \rho_{r e f} z_{0} e}$. Eq. (29) can be rewritten as

$$
\nabla^{2}\left(\bar{u}_{1}^{E}-\frac{\operatorname{Re} \cdot \bar{E}}{\bar{\mu}} \cdot \bar{\psi}\right)=0
$$

A new velocity is defined as

$$
\bar{u}_{1}^{E^{*}}=\bar{u}_{1}^{E}-\frac{\operatorname{Re} \cdot \bar{E}}{\bar{\mu}} \cdot \bar{\psi}
$$

Substituting Eq. (31) into Eq. (30), Eq. (30) transforms into

$$
\nabla^{2} \bar{u}_{1}^{E^{*}}=0
$$

In polar coordinates, Eq. (32) is specified as

$$
\frac{1}{r} \frac{\partial}{\partial r}\left(r \frac{\partial \bar{u}_{1}^{E^{*}}}{\partial r}\right)+\frac{1}{r^{2}} \frac{\partial^{2} \bar{u}_{1}^{E^{*}}}{\partial \theta^{2}}=0
$$

The solution of Eq. (33) can be described using the following equation

$$
\bar{u}_{1}^{E^{*}}=a_{10}^{E}+\sum_{j=1}^{J} r^{j}\left(a_{1 j}^{E} \cos (j \theta)+b_{1 j}^{E} \sin (j \theta)\right)
$$

From Eq. (31), the distribution of electroosmotic velocity is

$$
\bar{u}_{1}^{E}(r, \theta)=a_{10}^{E}+\frac{\operatorname{Re} \cdot \bar{E}}{\bar{\mu}} \cdot \bar{\psi}(r, \theta)+\sum_{j=1}^{J} r^{j}\left(a_{1 j}^{E} \cos (j \theta)+b_{1 j}^{E} \sin (j \theta)\right)
$$

The non-slip boundary conditions can be expressed as

$$
a_{10}^{E}+\sum_{j=1}^{J} r_{i}^{j}\left(a_{1 j}^{E} \cos \left(j \theta_{i}\right)+b_{1 j}^{E} \sin \left(j \theta_{i}\right)\right)=-\frac{\operatorname{Re} \cdot \bar{E}}{\bar{\mu}} \cdot \bar{\psi}\left(r_{i}, \theta_{i}\right)
$$

Using similar method, the electroosmotic velocity of fluid 2 is 


$$
\bar{u}_{2}^{E}=a_{20}^{E}+\sum_{j=1}^{J} r^{j}\left(a_{2 j}^{E} \cos (j \theta)+b_{2 j}^{E} \sin (j \theta)\right)
$$

The non-slip boundary conditions can be defined as

$$
a_{20}^{E}+\sum_{j=1}^{J} r_{i}^{j}\left(a_{2 j}^{E} \cos (j \theta)+b_{2 j}^{E} \sin (j \theta)\right)=0
$$

The matching conditions at the interface can be described as

$$
\left\{\begin{array}{l}
\bar{u}_{1}^{E}\left(r_{i}, \theta_{i}\right)=\bar{u}_{2}^{E}\left(r_{i}, \theta_{i}\right) \\
\frac{\partial \bar{u}_{1}^{E}\left(r_{i}, \theta_{i}\right)}{\partial n}=\beta \frac{\partial \bar{u}_{2}^{E}\left(r_{i}, \theta_{i}\right)}{\partial n}-M \bar{\rho}_{q}^{s}
\end{array}\right.
$$

where $M$ is the electrokinetic effect in the matching conditions, $M=\frac{\varepsilon_{0} \varepsilon_{r} k_{b} T E}{z_{0} e U_{r e f} \mu_{r e f}}, \bar{\rho}_{q}^{s}$ is the density of surface charge at the interface, $\bar{\rho}_{q}^{s}=-\frac{\partial \bar{\psi}}{\partial n}$. From Eqs.(36), and (38), the non-slip boundary conditions can be described using the following equations

$$
\left\{\begin{array}{rr}
a_{10}^{E}+0 \cdot a_{20}^{E}+\sum_{j=1}^{J} r_{i}^{j}\left(a_{1 j}^{E} \cos \left(j \theta_{i}\right)+b_{1 j}^{E} \sin \left(j \theta_{i}\right)+0 \cdot a_{2 j}^{E}+0 \cdot b_{2 j}^{E}\right)=-\frac{\mathrm{Re} \cdot \bar{E}}{\bar{\mu}} \cdot \bar{\psi}\left(r_{i}, \theta_{i}\right) \\
& \text { (for fluid 1) } \\
0 \cdot a_{10}^{E}+a_{20}^{E}+\sum_{j=1}^{J} r_{i}^{j}\left(0 \cdot a_{1 j}^{E}+0 \cdot b_{1 j}^{E}+a_{2 j}^{E} \cos (j \theta)+b_{2 j}^{E} \sin (j \theta)\right)=0 & \text { (for fluid 2) }
\end{array}\right.
$$

The matching conditions of Eq. (39) can be described as

$$
\begin{gathered}
a_{10}^{E}-a_{20}^{E}+\sum_{j=1}^{J}\left[r_{i}^{j} \cos \left(j \theta_{i}\right)+C_{1}^{E}(j)\right] a_{1 j}^{E}+\left[r_{i}^{j} \sin \left(j \theta_{I i}\right)+S_{1}^{E}(j)\right] b_{1 j}^{E} \\
\left.=-\frac{\operatorname{Re} \cdot \bar{E}}{\bar{\mu}} \cdot \bar{\psi}\left(r_{i}, \theta_{i}\right)+M C_{2}^{E}(j)\right]-\left[r_{i}^{j} \sin \left(j \theta_{I i}\right)+\beta S_{2}^{E}(j)\right] b_{2 j}^{E}-\frac{\operatorname{Re} \cdot \bar{E}}{\bar{\mu}_{1}} \cdot \frac{\partial \bar{\psi}\left(r_{i}, \theta_{i}\right)}{\partial n}
\end{gathered}
$$

where $C_{1}^{E}(j), S_{1}^{E}(j), C_{2}^{E}(j)$ and $S_{2}^{E}(j)$ can be described as

$$
\begin{aligned}
& C_{1}^{E}(j)=C_{2}^{E}(j)=r_{i}^{j-1} \cdot \cos \left(j \theta_{i}\right) \cdot j \cdot \cos \theta_{i}+r_{i}^{(j-2)} \cdot \sin \left(j \theta_{i}\right) \cdot j \cdot \sin \theta_{i} \\
& S_{1}^{E}(j)=S_{2}^{E}(j)=r_{i}^{j-1} \cdot \sin \left(j \theta_{i}\right) \cdot j \cdot \cos \theta_{i}-r_{i}^{(j-2)} \cdot \cos \left(j \theta_{i}\right) \cdot j \cdot \sin \theta_{i}
\end{aligned}
$$

Similar to the velocity driven by pressure gradient, the real coefficients of $a_{10}^{E}, a_{20}^{E}$, $a_{1 j}^{E}, b_{1 j}^{E}, a_{2 j}^{E}$, and $b_{2 j}^{E}$ in Eq. (40) can also be calculated using the method in Appendix A and Appendix D, and the velocity profile driven by electroosmotic force can be described using Eqs. (42) and (43). 


\subsection{Volume flow rates}

The dimensionless volumetric flowrates through the rectangular-cross-section channel can be defined as $\bar{q}_{1}=\bar{q}_{1}^{E}+\bar{q}_{1}^{p}=q_{1} /\left(L_{r e f}^{2} U_{r e f}\right)$ and $\bar{q}_{2}=\bar{q}_{2}^{E}+\bar{q}_{2}^{p}=q_{3} /\left(L_{r e f}^{2} U_{r e f}\right)$

The dimensionless flowrates are given as

$$
\begin{aligned}
& \bar{q}_{1}^{E}=\iint_{S 1} \bar{u}_{1}^{E}(\bar{x}, \bar{y}) d S \\
& \bar{q}_{1}^{p}=\iint_{S 1} \bar{u}_{1}^{P}(\bar{x}, \bar{y}) d S \\
& \bar{q}_{2}^{E}=\iint_{S 2} \bar{u}_{2}^{E}(\bar{x}, \bar{y}) d S \\
& \bar{q}_{2}^{p}=\iint_{S 2} \bar{u}_{2}^{P}(x, \bar{y}) d S
\end{aligned}
$$

where the integration limits of $S_{1}$ and $S_{2}$ are the area of cross section of fluid 1 and fluid 2 respectively. Substituting $\bar{u}_{1}^{E}, \bar{u}_{1}^{p}, \bar{u}_{2}^{E}$, and $\bar{u}_{2}^{p}$ into Eqs. (44)-(47), the dimensionless volumetric flowrates can be calculated.

\section{Convergence and comparison with previous results}

Before the analysis of the results obtained via the analytical model, it is necessary to confirm the accuracy of the solution scheme. A convergence test has been performed to determine the accuracy of the solution with respect to the number of boundary $(\mathrm{N})$ and with respect to the highest order of Bessel' function (J).

Figure 4 (a) shows the detail comparisons of the fully developed velocity profile $(x=$ 0 ) obtained using various computational grids with the exact solution when $J=17$. The following parameters are used in the calculation: $K=13, \theta_{c}=90^{0}, h_{1}=0.5, h_{2}=0.5$, $\bar{\xi}_{1}=\bar{\xi}_{2}=\bar{\xi}_{4}=1, \bar{\xi}_{3}=0, \mu_{1}=\mu_{2}$. The results presented in Fig. 4 (a) show the decay of discretization error with increased the total grid number N. It can be seen that the exact solution is reproduced accurately. Fig. 4(b) shows the comparisons of the fully developed velocity profiles obtained using four values of $\mathrm{J}$ with the exact solution when the total special boundary condition number $\mathrm{N}=280$. $\mathrm{J}$ is the highest order of modified Bessel 
functions. Under the same total number of boundary condition $\mathrm{N}$, a large value of $\mathrm{J}$ corresponds to a smaller error of calculation, the velocity profile becomes more accurate.

From all of above results, we can conclude that the interface between the two fluids is well captured and this semi-analytical method is suitable. In the following simulations, $\mathrm{N}=280$ and $\mathrm{J}=17$ are used.

The validity of the analytical model with curved interface has been checked by comparing with experimental results available in the literature. For the comparison, the interface is flat $\left(h_{\mathrm{s}}=0, h_{1}=0.5, h_{2}=0.5, \theta_{c}=90^{\circ}\right), \mathrm{N}=240$ and $\mathrm{J}=17$. Comparison between the analytical solution of the model presented in this paper and the experimental results of Wang et al [8] at the symmetric line $(x=0)$ of a rectangular microchannel. Our results agree well with the published experimental data.

\section{Results and discussion}

\subsection{Electric potential}

Figs $6(a, b, c)$ show the 2-D electric potential distributions in the high electric mobility fluid 1 for the concave interface $\left(h_{\mathrm{s}}=0.2, \theta_{c}=50^{0}\right)$, the plane interface $\left(h_{\mathrm{s}}=0\right.$, $\left.\theta_{c}=90^{\circ}\right)$ and the convex interface $\left(h_{\mathrm{s}}=-0.2, \theta_{c}=130^{\circ}\right)$, respectively. While Fig 7 shows the corresponding electric potential at the symmetric line of the rectangular microchannel. The other parameters are $K=13, h_{1}=0.5-h_{\mathrm{s}}, h_{2}=0.5, \bar{\xi}_{1}=\bar{\xi}_{2}=\bar{\xi}_{3}=\bar{\xi}_{4}=1$, $\bar{w}=0.5, \bar{h}=1, \mathrm{~N}=240$ and $\mathrm{J}=17$.

The results indicate that the interface shape influences the electric potential distribution of the two-fluid flow in the rectangular microchannel. Before the semianalytical approach is employed on rectangular microchannels with curved interfaces, the proposed approach is compared with the analytical solution of the plane interface proposed by Gao et al [14]. Fig. 7 shows the comparison between analytical solution [14] and the semi-analytical solution for the two-fluid electroosmotic flow in rectangular microchannels. It is clearly shown that the solutions are identical.

When two interfaces (wall and liquid interface) are sufficiently close to each other, the electrical double layers will interact with each other [30]. The effects of the EDL interaction are important to the electrokinetics flows within the microchannel. To 
investigate the effects of interface shape on the electric potential and velocity distributions, we fix the liquid faction of the two-fluid. The liquid fractions of fluid 1 and fluid 2 are defined as follows:

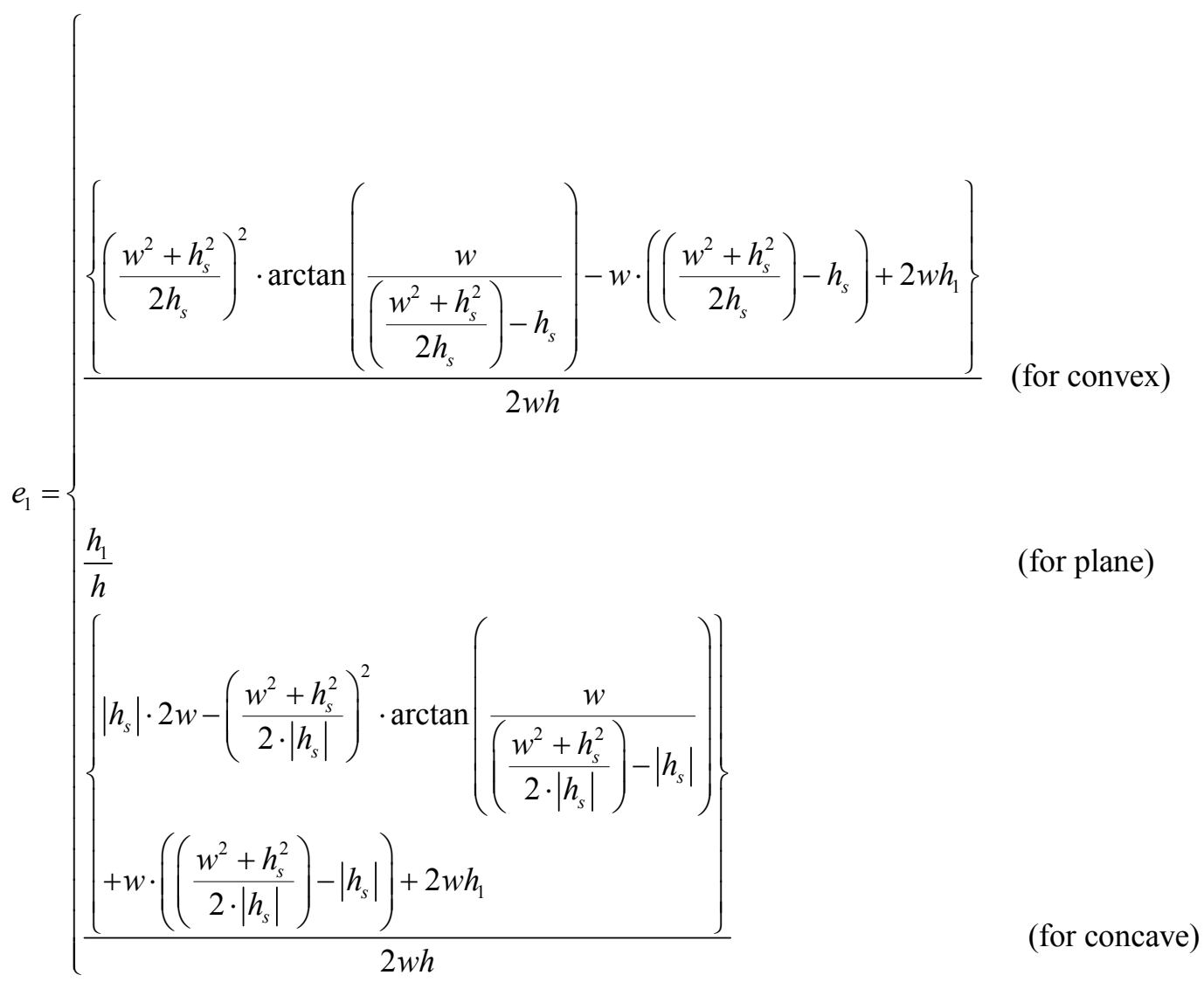

and

$$
e_{2}=1-e_{1}
$$

where $e_{1}$ and $e_{2}$ are the liquid fractions of fluid 1 and fluid 2, $\left|h_{s}\right|$ is the absolute value of interface height measured from the origin. In the following calculation, the liquid fractions are $e_{1}=e_{2}=0.5$. When $h_{s}$ is fixed, $h_{1}$ can be calculated using Eq. (48), and $\bar{h}_{2}=1-\bar{h}_{s}-\bar{h}_{1}$.

Fig. 8 shows the interface shapes of the concave, plane and the convex interfaces at the same liquid fraction of 0.5 . Symmetric section $a-a$, section $b-b$ and section $c-c$ are indicated in Fig 8. Sections a-a, b-b and c-c are located at $x=0, x=0.3$ and $x=0.4$ respectively. The potential and velocity distributions in these sections will be investigated. 
Fig. $9(a, b, c)$ show the comparisons of the electric potential distributions for the different interfaces: concave, plane and the convex interfaces at the same liquid fraction of various sections $\mathrm{a}-\mathrm{a}, \mathrm{b}-\mathrm{b}$ and $\mathrm{c}-\mathrm{c}$ respectively. In the calculation, $h_{\mathrm{s}}=0.2$ for concave interface, $h_{\mathrm{s}}=0$ for plane interface, and $h_{\mathrm{s}}=-0.2$ for convex interface, $K=13, h_{1}=0.5$ $h_{\mathrm{s}}, \bar{\xi}_{1}=\bar{\xi}_{2}=\bar{\xi}_{3}=\bar{\xi}_{4}=1, \bar{w}=0.5, \bar{h}=1, \mathrm{~N}=240$ and $\mathrm{J}=17, e_{1}=e_{2}=0.5, h_{1}$ can be calculated using Eq. (48), and then $\bar{h}_{2}=1-\bar{h}_{s}-\bar{h}_{1}$. Due to surface charges EDLs form at the liquid-liquid interface and channel walls that are in contact with the conducting fluid 1. In general, the electric potential decreases rapidly from the channel wall / liquid-liquid interface to the bulk region of the conducting liquid. The results show that the electric potential and the net charge density are highest for the concave interface due to the closest distance between liquid-liquid interface and the channel walls. The concave interface exhibits sharp corner (region A in Fig. 6) at the liquid-wall interface where the EDL overlaps (the calculated EDL thickness is $\frac{1}{\kappa} \approx 100 \mathrm{~nm}$ ). This overlapped EDL results in higher values of the electric potential and net charge density.

\subsection{Velocity distributions and flow rates}

From Eq. (10), the velocity $u$ of two-fluid can be decomposed into two parts, $u^{p}$ and $u^{E} \cdot u^{p}$ corresponds to velocity driven by pressure gradient and $u^{E}$ corresponds to velocity driven by electroosmotic effect. To investigate the influences of interface shapes on the velocity distributions, we analyze the following cases: (1) Two-fluid electroosmotic flow, $u^{E}$ with zero pressure gradient; (2) The two-fluid is driven by the combined electroosmotic force and pressure gradient.

\section{$\underline{\text { Two fluid electroosmotic flows }}$}

With zero pressure gradient is applied across the microchannel, the flow is mainly a two-fluid electroosmotic flow.

Fig. $10(\mathrm{a}, \mathrm{b}, \mathrm{c})$ show the comparison of the dimensionless velocity profiles, $u^{E}$ for the concave interface, plane interface and convex interface at sections $a-a, b-b, c-c$ respectively. In the simulation, we fix the liquid fraction $\left(e_{1}=e_{2}=0.5\right)$ of the two fluid 
and the applied electric field $E_{\mathrm{x}}=1000, \bar{\mu}_{1}=\bar{\mu}_{2}=1, \bar{\rho}_{1}=\bar{\rho}_{2}=1$, the other parameters are same with section 4.1 .

When electric fields are applied across the conducting fluids, the conducting fluids 1 is driven by electroosmosis, which drags the non conducting fluid 2 by the hydrodynamic shear force. By this way, the non conducting fluid can be delivered by electroosmosis. Comparison of the velocity distributions at different sections ( $a-a, b-b$ and $c-c)$ of the graphs shows that the concave interface produces the highest electroosmotic velocity. This is due to the larger values of the electric potential and net charge density induced by the concave interface. The velocity profile of the non conducting fluid is passive.

Fig 11 shows the influences of the interface shape on the dimensionless volumetric flow rate. For a given liquid fraction $\left(e_{1}=e_{2}=0.5\right)$ and applied electric filed $E_{\mathrm{x}}=1000$, the volumetric flow rates of the conducting fluids increase with the decrease in contact angle $\left(\theta_{c}\right)$.

The results show that the volumetric electroosmotic flow rate of the conducting liquid with the concave interface it is about 1.5 times larger than that of plane interface when the contact angle is about $0^{\circ}$ which is due to the larger values of the electric potential and hence higher electroosmotic velocity.

\section{Two-fluid flow driven by electroosmosis and pressure gradient}

When both pressure gradient and electric field are applied, the flow is driven by electroosmotic body force and pressure gradient. In the analysis, we fix the liquid fraction $\left(e_{1}=e_{2}=0.5\right)$, electric field $E_{\mathrm{x}}=1000$, pressure gradient $d \bar{p} / d \bar{z}=50000$, and viscosities $\bar{\mu}_{1}=\bar{\mu}_{2}=1$.

Fig. 12(a, b, c) show the comparison of the dimensionless velocity profiles, $u$, for the concave interface, plane interface and convex interface at section $a-a, b-b, c-c$ respectively. The other parameters are same with section 4.1. The result shows that the velocity profile, $\bar{u}$ is the superposition of the solutions of the electroosmotic velocity profile $\bar{u}^{E}$ and the pressure-driven parabolic profile, $\bar{u}^{p}$.

As the same viscosity $\bar{\mu}_{1}=\bar{\mu}_{2}=1, \bar{u}^{p}$ is in face a pressure driven flow of single fluid, and exhibits a parabolic velocity profile dimensionless pressure driven velocity profiles. 
The dimensionless pressure driven velocity profiles, $u^{p}$, at different cross section $(\mathrm{a}-\mathrm{a}, \mathrm{b}-$ b, c-c) show that near the wall (section c-c) the velocity is affected by the non-slip boundary condition hence resulted in a lower velocity. The results also show $u^{p}$ is independent of the interface shapes when $\bar{\mu}_{1}=\bar{\mu}_{2}=1$.

Comparison of the velocity profiles at different cross sections a-a and c-c illustrate that at cross section a-a, the pressure driven velocity $\bar{u}^{p}$ is distinctly larger than electroosmotic velocity $\bar{u}^{E}$. At this bulk two-fluid section a-a (region far away from EDL and the side wall), the fluid is influenced by (i) the faster lamina fluid layer immediately closer to the center due to the applied pressure gradient and (ii) the interfacial shear stress. Hence $\bar{u}^{p}$ dominates.

At cross section c-c, the fluid is influenced by the no-slip boundary condition and the viscous shear diffusion due to (i) the faster lamina fluid layers at the EDL region and (ii) the faster lamina fluid layers immediately closer to the center. Hence in this section the electroosmosis effect dominates.

The result also shows that the velocity profile, $\bar{u}$ is the superposition of the solutions of the electroosmotic velocity profile $\bar{u}^{E}$ and the pressure-driven parabolic profile, $\bar{u}^{p}$.

\section{Conclusions}

This paper presents a semi analytical model of the pressure driven two-fluid flow in rectangular microchannels with electroosmotic effect. Different from the previous work, the curved interface between the immiscible fluids are used to replace the assumed plane interface.

The validity of the semi-analytical model with curved interfaces has been checked by comparing with the solution obtained with the results available in the literatures. For special condition of plane interface, the distribution of electric potential, and the profiles of pressure driven velocity and electroosmotic velocity have good agreements with the results of previous works.

The current work focuses on the effect of curved interface to the two-fluid flow in microchannel. The results show that the interface shapes influence the characteristics of 
the flow significantly. Especially in the sharp corner between interface and the wall, the electric potential is higher. Comparing with other cases, the concave model has highest electric potential under same boundary conditions. The velocity is also higher for concave model. Under same liquid fraction, the maximum volumetric flow rate of conducting fluid for concave is about 1.5 times of plane model.

\section{Appendix A}

In the following appendix, the least square method for numerical calculate can be introduced.

Eqs. (9), (22), and (40) can be described using matrix form as follows

$$
\mathbf{M B}=\mathbf{Z}
$$

In Eq. (50), $\mathbf{M}$ is the matrix for coefficients, $\mathbf{B}$ is the matrix for unknown parameters, $\mathbf{Z}$ is the matrix for boundaries. The parameter of $\mathbf{B}$ can be calculated numerically using the least-squares method that minimizes of $\|\mathbf{M B}-\mathbf{Z}\|$ is employed to find the optimal values of $\mathbf{B}$. The detail of $\mathbf{M}, \mathbf{B}$, and $\mathbf{Z}$ for Eqs. (9), (22), and (40) are shown in Appendix B, Appendix C, and Appendix D respectively.

\section{Appendix B}

In the following appendix, the details of $\mathbf{M}, \mathbf{B}$, and $\mathbf{Z}$ for Eqs. (9) are shown:

$$
\begin{gathered}
\mathbf{M}=\left(\begin{array}{ccc}
I_{0}\left(K r_{1}\right), & & I_{J}\left(K r_{1}\right) \cos \left(J \cdot \theta_{1}\right), I_{J}\left(K r_{1}\right) \sin \left(J \cdot \theta_{1}\right) \\
I_{0}\left(K r_{2}\right), & \cdots & I_{J}\left(K r_{2}\right) \cos \left(J \cdot \theta_{2}\right), I_{J}\left(K r_{2}\right) \sin \left(J \cdot \theta_{2}\right) \\
\vdots & \ddots & \vdots \\
I_{0}\left(K r_{N-1}\right), & \ldots & I_{J}\left(K r_{N-1}\right) \cos \left(J \cdot \theta_{N-1}\right), I_{J}\left(K r_{N-1}\right) \sin \left(J \cdot \theta_{N-1}\right) \\
I_{0}\left(K r_{N}\right), & & I_{J}\left(K r_{N}\right) \cos \left(J \cdot \theta_{N}\right), I_{J}\left(K r_{N}\right) \sin \left(J \cdot \theta_{N}\right)
\end{array}\right)_{N \times(2 J+1)} \\
\mathbf{B}=\left(\begin{array}{c}
A_{10} \\
\vdots \\
A_{1 J} \\
A_{2 J}
\end{array}\right)_{(2 J+1) \times 1}
\end{gathered}
$$




$$
\mathbf{Z}=\left(\begin{array}{l}
\bar{\psi}\left(r_{1}, \theta_{1}\right) \\
\bar{\psi}\left(r_{2}, \theta_{2}\right) \\
\vdots \\
\bar{\psi}\left(r_{N-1}, \theta_{N-1}\right) \\
\bar{\psi}\left(r_{N}, \theta_{N}\right)
\end{array}\right)_{N \times 1}
$$

where $\mathrm{N}$ is the total number of the boundary conditions and $J$ is the highest order of the Bessel functions chosen in this paper.

\section{Appendix C}

In the following appendix, the details of $\mathbf{M}, \mathbf{B}$, and $\mathbf{Z}$ for Eqs. (22) are shown:

$$
\begin{aligned}
& \mathbf{M}=\left(\begin{array}{l}
\mathbf{M}_{11} \\
\mathbf{M}_{22} \\
\mathbf{M}_{33}
\end{array}\right) \\
& \mathbf{M}_{11}=\left(\begin{array}{ccc}
1,0, r_{1}^{1} \cos \left(\theta_{1}\right), r_{1}^{1} \sin \left(\theta_{1}\right), & & r_{1}^{J} \cos \left(J \cdot \theta_{1}\right), r_{1}^{J} \sin \left(J \cdot \theta_{1}\right), 0,0 \\
1,0, r_{2}^{1} \cos \left(\theta_{2}\right), r_{2}^{1} \sin \left(\theta_{2}\right), & \cdots & r_{2}^{J} \cos \left(J \cdot \theta_{2}\right), r_{2}^{J} \sin \left(J \cdot \theta_{2}\right), 0,0 \\
\vdots & \ddots & \vdots \\
1,0, r_{N_{1}-1}^{1} \cos \left(\theta_{N_{1}-1}\right), r_{N_{1}-1}^{1} \sin \left(\theta_{N_{1}-1}\right), & \ldots & r_{N_{1}-1}^{J} \cos \left(J \cdot \theta_{N_{1}-1}\right), r_{N_{1}-1}^{J} \sin \left(J \cdot \theta_{N_{1}-1}\right), 0,0 \\
1,0, r_{N_{1}}^{1} \cos \left(\theta_{N_{1}}\right), r_{N_{1}}^{1} \sin \left(\theta_{N_{1}}\right), & & r_{N_{1}}^{J} \cos \left(J \cdot \theta_{N_{1}}\right), r_{N_{1}}^{J} \sin \left(J \cdot \theta_{N_{1}}\right), 0,0
\end{array}\right)_{N_{1} \times(4 J+2)} \\
& \mathbf{M}_{22}=\left(\begin{array}{ccc}
0,1,0,0, r_{1}^{1} \cos \left(\theta_{1}\right), r_{1}^{1} \sin \left(\theta_{1}\right), & & r_{1}^{J} \cos \left(J \cdot \theta_{1}\right), r_{1}^{J} \sin \left(J \cdot \theta_{1}\right) \\
0,1,0,0, r_{2}^{1} \cos \left(\theta_{2}\right), r_{2}^{1} \sin \left(\theta_{2}\right), & \cdots & r_{2}^{J} \cos \left(J \cdot \theta_{2}\right), r_{2}^{J} \sin \left(J \cdot \theta_{2}\right) \\
\vdots & \ddots & \vdots \\
0,1,0,0, r_{N_{2}-1}^{1} \cos \left(\theta_{N_{2}-1}\right), r_{N_{2}-1}^{1} \sin \left(\theta_{N_{2}-1}\right), & \ldots & r_{N_{2}-1}^{J} \cos \left(J \cdot \theta_{N_{2}-1}\right), r_{N_{2}-1}^{J} \sin \left(J \cdot \theta_{N_{2}-1}\right) \\
0,1,0,0, r_{N_{2}}^{1} \cos \left(\theta_{N_{2}}\right), r_{N_{2}}^{1} \sin \left(\theta_{N_{2}}\right), & & r_{N_{2}}^{J} \cos \left(J \cdot \theta_{N_{2}}\right), r_{N_{2}}^{J} \sin \left(J \cdot \theta_{N_{2}}\right)
\end{array}\right)_{N_{2} \times(4 J+2)} \\
& \mathbf{M}_{33}=\left(\begin{array}{ccc}
\beta,-1,\left[\beta r_{1}^{1} \cos \left(\theta_{1}\right)+C_{1}(1)\right], & & {\left[-r_{1}^{J} \cos \left(J \cdot \theta_{1}\right)-C_{1}(J)\right],\left[-r_{1}^{J} \sin \left(J \cdot \theta_{1}\right)-S_{1}(1)\right]} \\
\beta,-1,\left[\beta r_{2}^{1} \cos \left(\theta_{2}\right)+C_{1}(1)\right], & \cdots & {\left[-r_{2}^{J} \cos \left(J \cdot \theta_{2}\right)-C_{1}(J)\right],\left[-r_{2}^{J} \sin \left(J \cdot \theta_{2}\right)-S_{1}(1)\right]} \\
\vdots & \ddots & \vdots \\
\beta,-1,\left[\beta r_{N_{3}-1}^{1} \cos \left(\theta_{N_{3}-1}\right)+C_{1}(1)\right], & \ldots & {\left[-r_{N_{3}-1}^{J} \cos \left(J \cdot \theta_{N_{3}-1}\right)-C_{1}(J)\right],\left[-r_{N_{3}-1}^{J} \sin \left(J \cdot \theta_{N_{3}-1}\right)-S_{1}(1)\right]} \\
\beta,-1,\left[\beta r_{N_{3}}^{1} \cos \left(\theta_{N_{3}}\right)+C_{1}(1)\right], & & {\left[-r_{N_{3}}^{J} \cos \left(J \cdot \theta_{N_{3}}\right)-C_{1}(J)\right],\left[-r_{N_{3}}^{J} \sin \left(J \cdot \theta_{N_{3}}\right)-S_{1}(1)\right]}
\end{array}\right)_{N_{3} \times(4 J+2)} \\
& \mathbf{B}=\left(\begin{array}{l}
\mathbf{B}_{11} \\
\mathbf{B}_{21} \\
\mathbf{B}_{31}
\end{array}\right)
\end{aligned}
$$




$$
\begin{aligned}
& \mathbf{B}_{11}=\mathbf{B}_{22}=\mathbf{B}_{33}=\left(\begin{array}{c}
a_{10}^{p} \\
a_{20}^{p} \\
\vdots \\
a_{2 J}^{p} \\
b_{2 J}^{p}
\end{array}\right)_{(4 J+2) \times 1} \\
& \mathbf{Z}=\left(\begin{array}{l}
\mathbf{Z}_{11} \\
\mathbf{Z}_{22} \\
\mathbf{Z}_{33}
\end{array}\right) \\
& \mathbf{Z}_{11}=\left(\begin{array}{c}
\frac{r_{1}^{2}}{4} \\
\vdots \\
\frac{r_{N_{1}}^{2}}{4}
\end{array}\right)_{N_{1} \times 1} \\
& \mathbf{Z}_{22}=\left(\begin{array}{c}
\frac{r_{1}^{2}}{4} \\
\vdots \\
\frac{r_{N_{2}}^{2}}{4}
\end{array}\right)_{N_{2} \times 1} \\
& \mathbf{Z}_{33}=\left(\begin{array}{l}
(\beta-1) \frac{r_{1}^{2}}{4} \\
\vdots \\
(\beta-1) \frac{r_{N_{3}}^{2}}{4}
\end{array}\right)_{N_{3} \times 1}
\end{aligned}
$$

where $\mathrm{N}_{1}$ is the total number of the boundary condition for fluid $1, \mathrm{~N}_{2}$ is the total number of the boundary condition for fluid $2, \mathrm{~N}_{3}$ is the total number of the matching condition at the interface, and $J$ is the highest order of the Bessel functions chosen in this paper.

\section{Appendix D}

In the following appendix, the details of $\mathbf{M}, \mathbf{B}$, and $\mathbf{Z}$ for Eqs. (40) are shown:

$$
\mathbf{M}=\left(\begin{array}{l}
\mathbf{M}_{11} \\
\mathbf{M}_{22} \\
\mathbf{M}_{33}
\end{array}\right)
$$




$$
\begin{aligned}
& \mathbf{M}_{11}=\left(\begin{array}{ccc}
1,0, r_{1}^{1} \cos \left(\theta_{1}\right), r_{1}^{1} \sin \left(\theta_{1}\right), & & r_{1}^{J} \cos \left(J \cdot \theta_{1}\right), r_{1}^{J} \sin \left(J \cdot \theta_{1}\right), 0,0 \\
1,0, r_{2}^{1} \cos \left(\theta_{2}\right), r_{2}^{1} \sin \left(\theta_{2}\right), & \cdots & r_{2}^{J} \cos \left(J \cdot \theta_{2}\right), r_{2}^{J} \sin \left(J \cdot \theta_{2}\right), 0,0 \\
\vdots & \ddots & \vdots \\
1,0, r_{N_{1}-1}^{1} \cos \left(\theta_{N_{1}-1}\right), r_{N_{1}-1}^{1} \sin \left(\theta_{N_{1}-1}\right), & \ldots & r_{N_{1}-1}^{J} \cos \left(J \cdot \theta_{N_{1}-1}\right), r_{N_{1}-1}^{J} \sin \left(J \cdot \theta_{N_{1}-1}\right), 0,0 \\
1,0, r_{N_{1}}^{1} \cos \left(\theta_{N_{1}}\right), r_{N_{1}}^{1} \sin \left(\theta_{N_{1}}\right), & & r_{N_{1}}^{J} \cos \left(J \cdot \theta_{N_{1}}\right), r_{N_{1}}^{J} \sin \left(J \cdot \theta_{N_{1}}\right), 0,0
\end{array}\right)_{N_{1} \times(4 J+2)}
\end{aligned}
$$

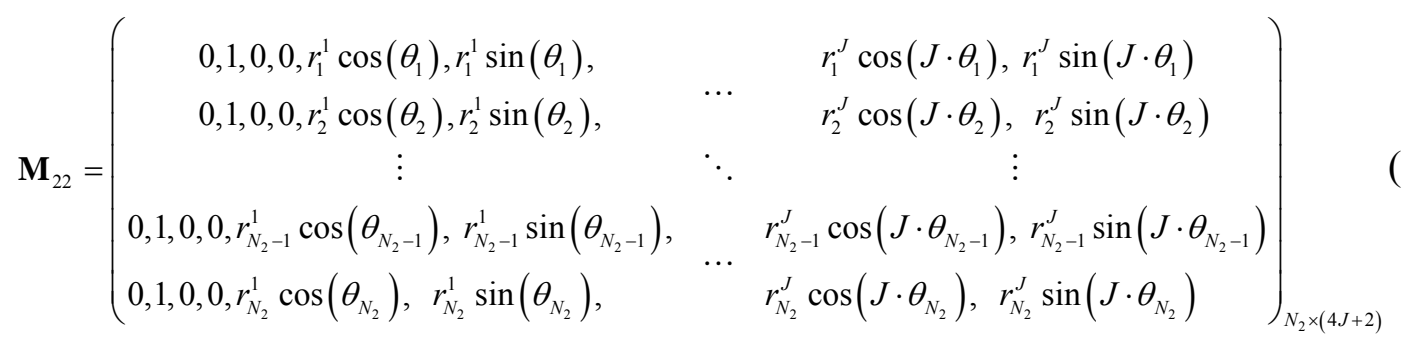

$$
\begin{aligned}
& \mathbf{M}_{33}=\left(\begin{array}{ccc}
1,-1,\left[r_{1}^{1} \cos \left(\theta_{1}\right)+C_{1}^{E}(1)\right], & & {\left[-r_{1}^{J} \sin \left(J \cdot \theta_{1}\right)-\beta S_{2}^{E}(J)\right]} \\
1,-1,\left[r_{2}^{1} \cos \left(\theta_{2}\right)+C_{1}^{E}(1)\right], & \cdots & {\left[-r_{2}^{J} \sin \left(J \cdot \theta_{2}\right)-\beta S_{2}^{E}(J)\right]} \\
\vdots & \ddots & \vdots \\
1,-1,\left[r_{N_{3}-1}^{1} \cos \left(\theta_{N_{3}-1}\right)+C_{1}^{E}(1)\right], & \ldots & {\left[-r_{N_{3}-1}^{J} \sin \left(J \cdot \theta_{N_{3}-1}\right)-\beta S_{2}^{E}(J)\right]} \\
1,-1,\left[r_{N_{3}}^{1} \cos \left(\theta_{N_{3}}\right)+C_{1}^{E}(1)\right], & & {\left[-r_{N_{3}}^{J} \sin \left(J \cdot \theta_{N_{3}}\right)-\beta S_{2}^{E}(J)\right]}
\end{array}\right)_{N_{3} \times(4 J+2)} \\
& \mathbf{B}=\left(\begin{array}{l}
\mathbf{B}_{11} \\
\mathbf{B}_{21} \\
\mathbf{B}_{31}
\end{array}\right) \\
& \mathbf{B}_{11}=\mathbf{B}_{22}=\mathbf{B}_{33}=\left(\begin{array}{c}
a_{10}^{E} \\
a_{20}^{E} \\
\vdots \\
a_{1 J}^{E} \\
B_{2 J}^{E}
\end{array}\right)_{(4 J+2) \times 1} \\
& \mathbf{Z}=\left(\begin{array}{l}
\mathbf{Z}_{11} \\
\mathbf{Z}_{22} \\
\mathbf{Z}_{33}
\end{array}\right) \\
& \mathbf{Z}_{11}=\left(\begin{array}{l}
-\frac{\operatorname{Re} \cdot \bar{E}}{\bar{\mu}} \cdot \bar{\psi}\left(r_{1}, \theta_{1}\right) \\
\vdots \\
-\frac{\operatorname{Re} \cdot \bar{E}}{\bar{\mu}} \cdot \bar{\psi}\left(r_{N_{1}}, \theta_{N_{1}}\right)
\end{array}\right)_{N_{1} \times 1}
\end{aligned}
$$




$$
\mathbf{Z}_{33}=\left(\begin{array}{l}
\mathbf{Z}_{22}=\left(\begin{array}{c}
0 \\
\vdots \\
0
\end{array}\right)_{N_{2} \times 1} \\
-\frac{\operatorname{Re} \cdot \bar{E}}{\bar{\mu}} \cdot \bar{\psi}\left(r_{1}, \theta_{1}\right)-M \bar{\rho}_{q}^{s}-\frac{\operatorname{Re} \cdot \bar{E}}{\bar{\mu}_{1}} \cdot \frac{\partial \bar{\psi}\left(r_{1}, \theta_{1}\right)}{\partial n} \\
\vdots \\
-\frac{\operatorname{Re} \cdot \bar{E}}{\bar{\mu}} \cdot \bar{\psi}\left(r_{N_{3}}, \theta_{N_{3}}\right)-M \bar{\rho}_{q}^{s}-\frac{\operatorname{Re} \cdot \bar{E}}{\bar{\mu}_{1}} \cdot \frac{\partial \bar{\psi}\left(r_{N_{3}}, \theta_{N_{3}}\right)}{\partial n}
\end{array}\right)_{N_{3} \times 1}
$$

where $\mathrm{N}_{1}$ is the total number of the boundary condition for fluid1, $\mathrm{N}_{2}$ is the total number of the boundary condition for fluid2, $\mathrm{N}_{3}$ is the total number of the matching condition for interface, and $J$ is the highest order of the Bessel functions chosen in this paper.

\section{References}

[1] G.M. Whitesides, A.D. Stroock, Flexible methods for microfluidics, Physics Today, 54(6) (2001) 42.

[2] D.R. Reyes, D. Iossifidis, P.A. Auroux, A. Manz, Micro total analysis systems. 1. Introduction, theory, and technology, Analytical Chemistry, 74(12) (2002) 2623-2636.

[3] H.A. Stone, A.D. Stroock, A. Ajdari, Engineering flows in small devices: Microfluidics toward a lab-on-a-chip, Annual Review of Fluid Mechanics, 36 (2004) 381-411.

[4] C.H. Chen, J.G. Santiago, A planar electroosmotic micropump, Journal of Microelectromechanical Systems, 11(6) (2002) 672-683.

[5] L.M. Fu, R.J. Yang, C.H. Lin, Y.J. Pan, G.B. Lee, Electrokinetically driven micro flow cytometers with integrated fiber optics for on-line cell/particle detection, Analytica Chimica Acta, 507(1) (2004) 163-169.

[6] A. Brask, G. Goranović, M.J. Jensen, H. Bruus, A novel electro-osmotic pump design for nonconducting liquids: Theoretical analysis of flow rate-pressure characteristics and stability, Journal of Micromechanics and Microengineering, 15(4) (2005) 883-891.

[7] D. Kohlheyer, G.A.J. Besselink, R.G.H. Lammertink, S. Schlautmann, S. Unnikrishnan, R.B.M. Schasfoort, Electro-osmotically controllable multi-flow microreactor, Microfluidics and Nanofluidics, 1(3) (2005) 242-248.

[8] C. Wang, Y. Gao, N.T. Nguyen, T.N. Wong, C. Yang, K.T. Ooi, Interface control of pressure-driven two-fluid flow in microchannels using electroosmosis, Journal of Micromechanics and Microengineering, 15(12) (2005) 2289-2297.

[9] R.J. Yang, C.C. Chang, S.B. Huang, G.B. Lee, A new focusing model and switching approach for electrokinetic flow inside microchannels, Journal of Micromechanics and Microengineering, 15(11) (2005) 2141-2148. 
[10] K.H. Kang, J. Park, I.S. Kang, K.Y. Huh, Initial growth of electrohydrodynamic instability of two-layered miscible fluids in T-shaped microchannels, International Journal of Heat and Mass Transfer, 49(23-24) (2006) 4577-4583.

[11] O. Ozen, N. Aubry, D.T. Papageorgiou, P.G. Petropoulos, Electrohydrodynamic linear stability of two immiscible fluids in channel flow, Electrochimica Acta, 51(25) (2006) 5316-5323.

[12] J.D. Zahn, V. Reddy, Two phase micromixing and analysis using electrohydrodynamic instabilities, Microfluidics and Nanofluidics, 2(5) (2006) 399-415.

[13] Y. Gao, T.N. Wong, C. Yang, T.O. Kim, Transient two-liquid electroosmotic flow with electric charges at the interface, Colloids and Surfaces A: Physicochemical and Engineering Aspects, 266(1-3) (2005) 117-128.

[14] Y. Gao, C. Wang, T.N. Wong, C. Yang, N.T. Nguyen, K.T. Ooi, Electro-osmotic control of the interface position of two-liquid flow through a microchannel, Journal of Micromechanics and Microengineering, 17(2) (2007) 358-366.

[15] H. Li, T.N. Wong, N.T. Nguyen, Electroosmotic control of width and position of liquid streams in hydrodynamic focusing, Microfluidics and Nanofluidics, (2009) 1-9.

[16] G.B. Lee, B.H. Hwei, G.R. Huang, Micromachined pre-focused $\mathrm{M} \times \mathrm{N}$ flow switches for continuous multi-sample injection, Journal of Micromechanics and Microengineering, 11(6) (2001b) 654-661.

[17] Y. Gao, T.N. Wong, C. Yang, K.T. Ooi, Two-fluid electroosmotic flow in microchannels, J. Colloid Interface Sci., 284(1) (2005b) 306-314.

[18] H. Li, T.N. Wong, N.T. Nguyen, Sample flow switching technique based on combined effect of hydrodynamic and electroosmosis, in, 2010, pp. 59-63.

[19] M. Bentwich, Two-phase axial laminar flow in a pipe with naturally curved interface, Chemical Engineering Science, 31(1) (1976) 71-76.

[20] J. Ong, G. Enden, A.S. Popel, Converging three-dimensional stokes flow of two fluids in a T-type bifurcation, Journal of Fluid Mechanics, 270 (1994) 51-71.

[21] N. Brauner, J. Rovinsky, D.M. Maron, Analytical solution for laminar-laminar twophase stratified flow in circular conduits, Chemical Engineering Communications, 141142 (1996) 103-143.

[22] N. Brauner, J. Rovinsky, D. Moalem Maron, Determination of the interface curvature in stratified two-phase systems by energy considerations, International Journal of Multiphase Flow, 22(6) (1996) 1167-1185.

[23] N. Brauner, D. Moalem Maron, J. Rovinsky, A two-fluid model for stratified flows with curved interfaces, International Journal of Multiphase Flow, 24(6) (1998) 975-1004.

[24] D. Gorelik, N. Brauner, The interface configuration in two-phase stratified pipe flows, International Journal of Multiphase Flow, 25(6-7) (1999) 977-1007.

[25] T.S. Ng, C.J. Lawrence, G.F. Hewitt, Interface shapes for two-phase laminar stratified flow in a circular pipe, International Journal of Multiphase Flow, 27(7) (2001) $1301-1311$.

[26] T.S. Ng, C.J. Lawrence, G.F. Hewitt, Laminar stratified pipe flow, International Journal of Multiphase Flow, 28(6) (2002) 963-996.

[27] G. Goranović, Electrohydrodynamic aspects of two fluid microfluidic systems: theory and simulation, Technical University of Denmark, 2003. 
[28] R.J. Hunter, Zeta Potential in Colloid Science: Principles and Applications, Harcourt Brace Jovanovich, London San Diego New York Berkely Boston Sydney Tokyo Toronto, 1981.

[29] R.G. Cox, T.G.M. Van De Ven, Electroviscous forces on a charged particle suspended in a flowing liquid, J. Fluid Mech., 338 (1997) 1-34.

[30] Y. Zhang, T.N. Wong, C. Yang, K.T. Ooi, Electroosmotic flow in irregular shape microchannels, International Journal of Engineering Science, 43(19-20) (2005) 14501463.

[31] W.M. Deen, Analysis of Transport Phenomena, Oxford university press, New York Oxford, 1998. 


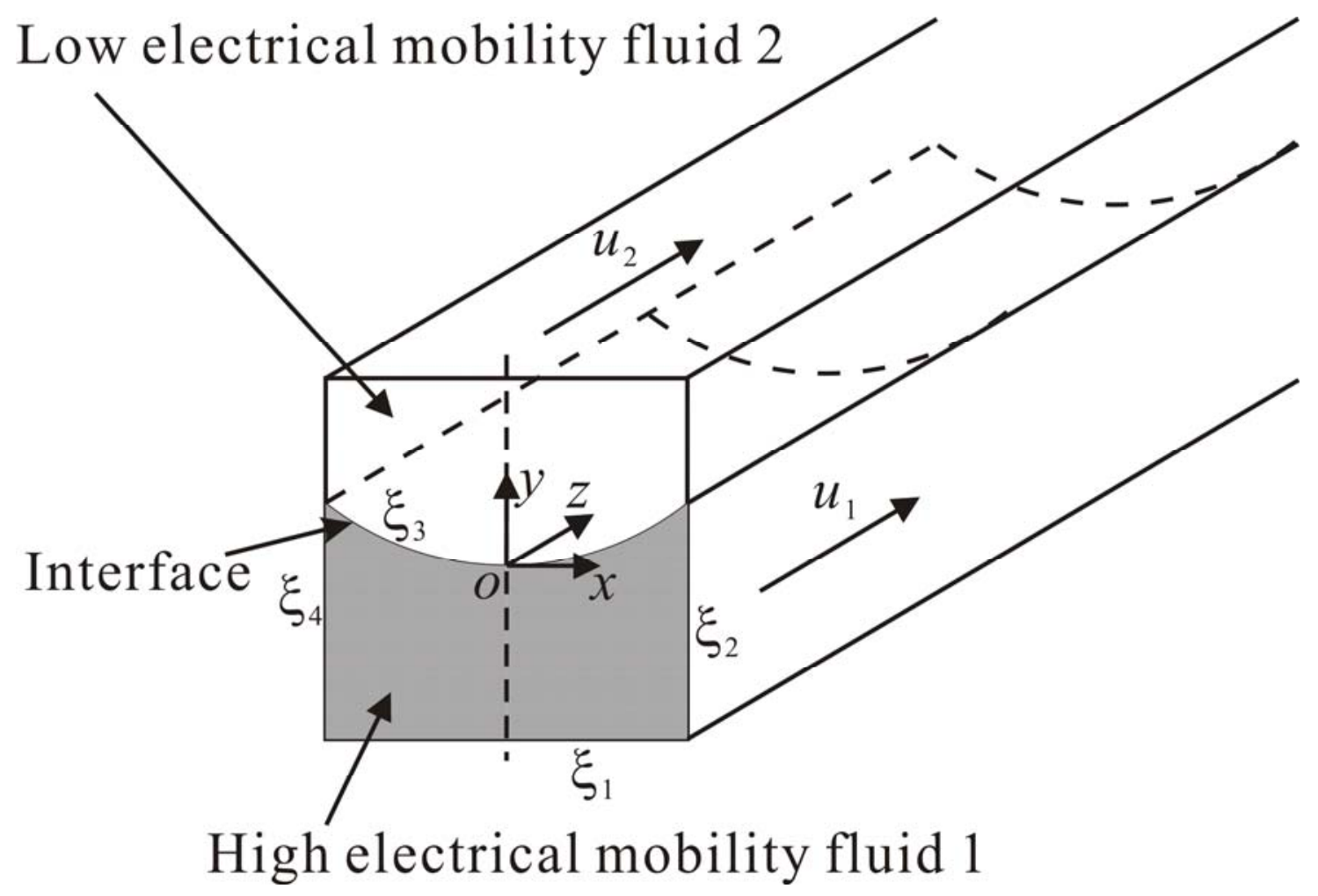

Fig. 1 Schematic of the cross section of two-fluid flow in microchannel with curved interface and coordinate system 


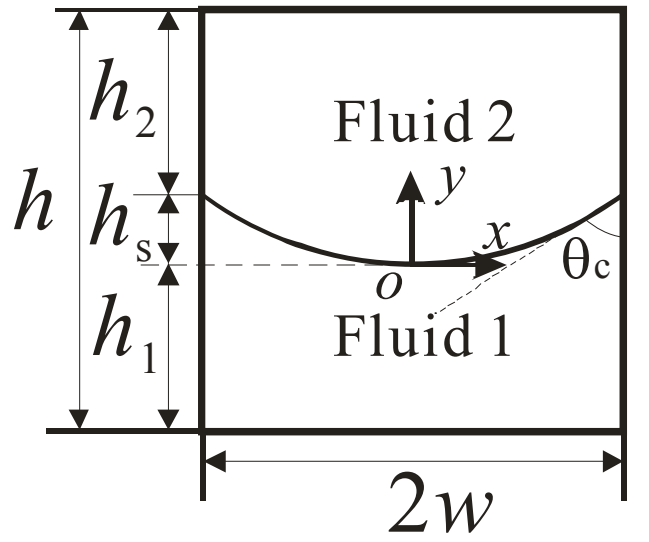

(a) Concave $\left(\theta_{\mathrm{c}}<90^{\circ}, h_{\mathrm{s}}>0\right)$

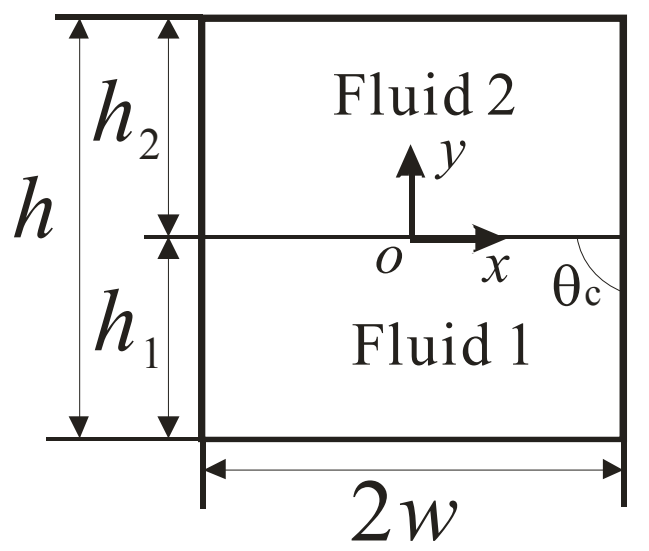

(b) Plane $\left(\theta_{\mathrm{c}}=90^{\circ}, h_{\mathrm{s}}=0\right)$

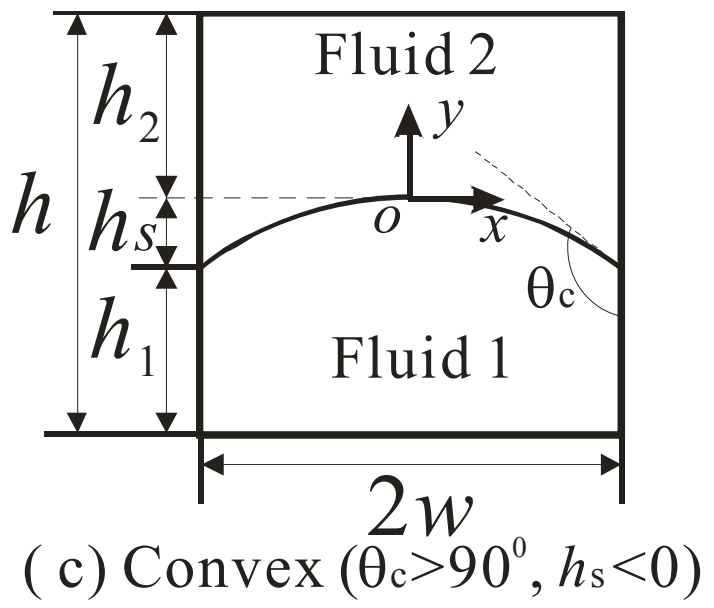

Fig. 2 Various interface shapes in a rectangular microchannel 


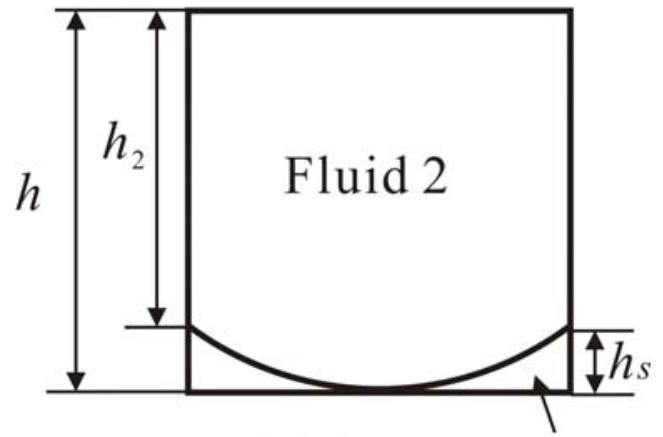

(a) $h_{1}=0 \quad$ Fluid 1
Fluid 2

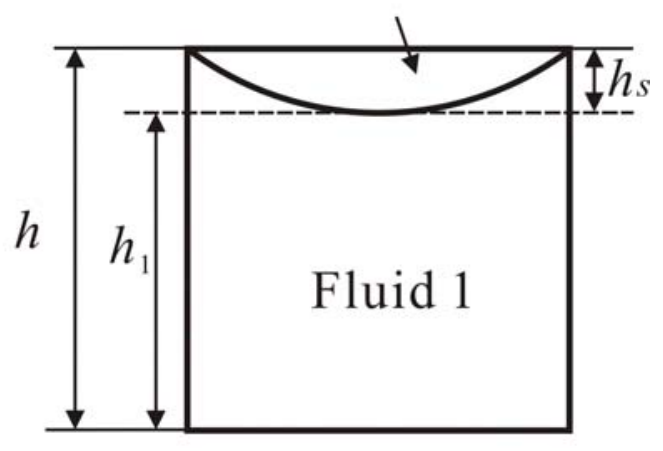

(b) $h_{2}=0$

Fig. 3 The limitations of the interface location 


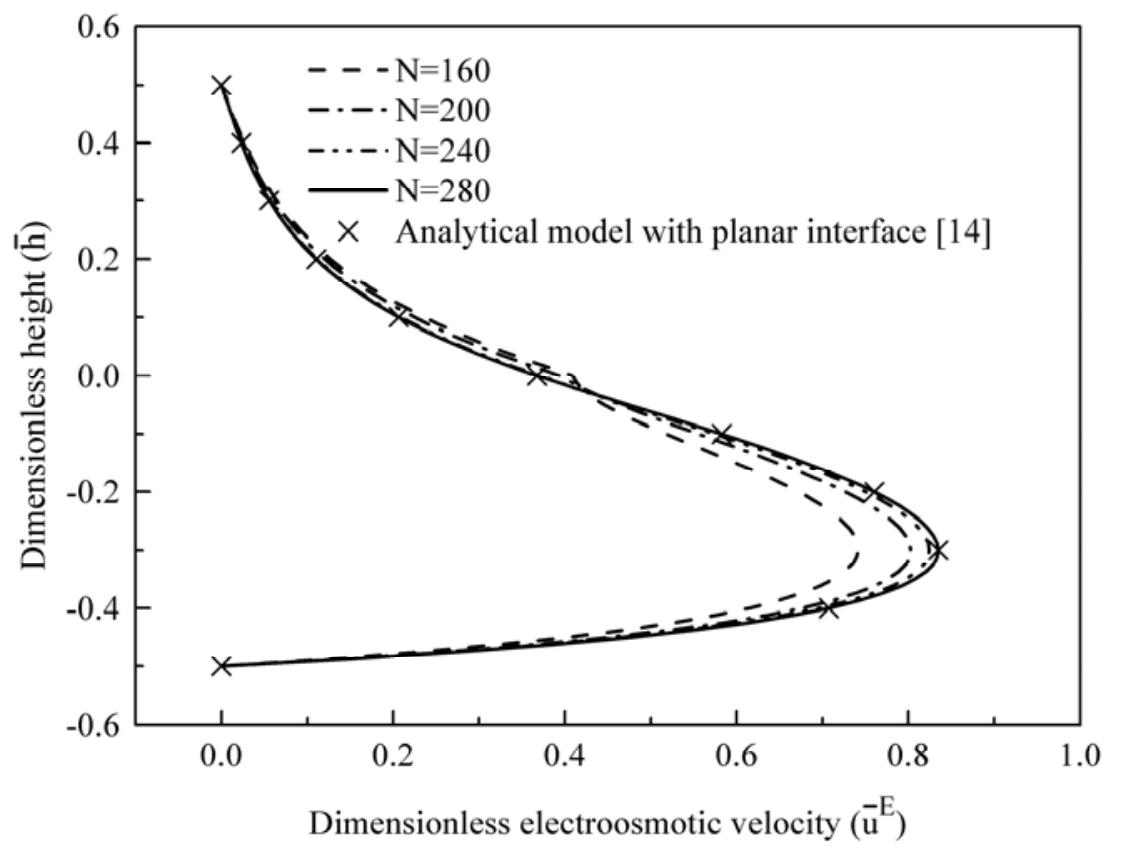

(a)

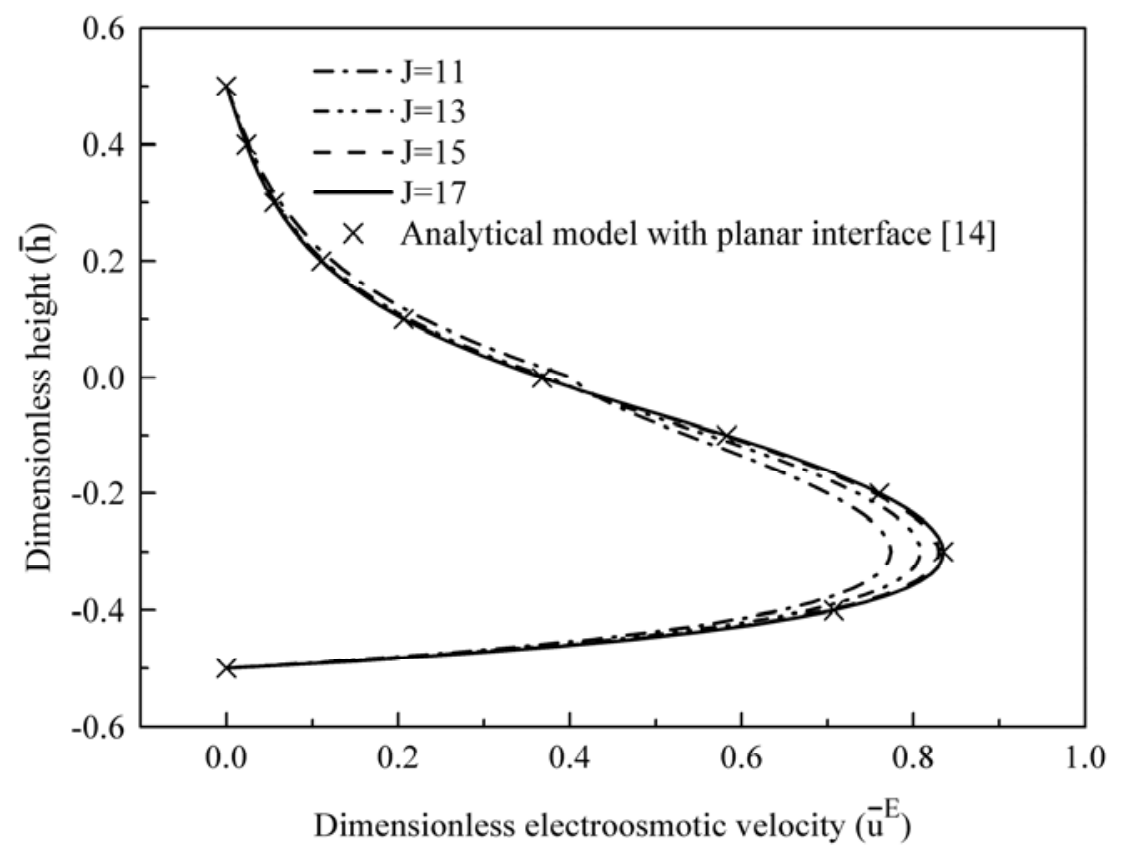

(b)

Fig. 4 Electroosmotic velocity profiles for various value of $\mathrm{N}$ and $\mathrm{J}$

(a) Various $\mathrm{N}$ and fixed $\mathrm{J}=17$

(b) Various $\mathrm{J}$ and fixed $\mathrm{N}=280$ 


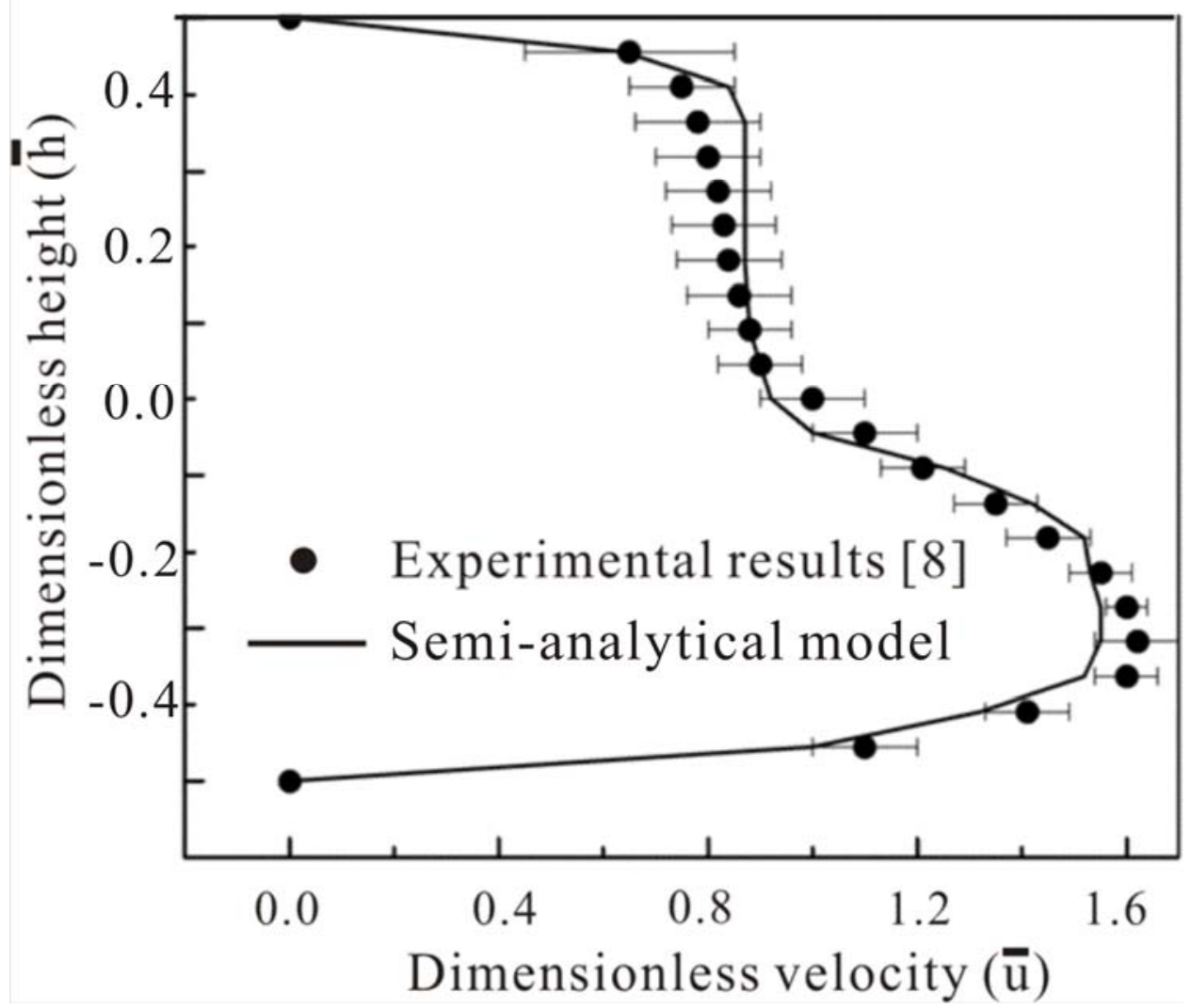

Fig. 5 Comparison of the velocity profile between the semi-analytical model and experimental data [8] 


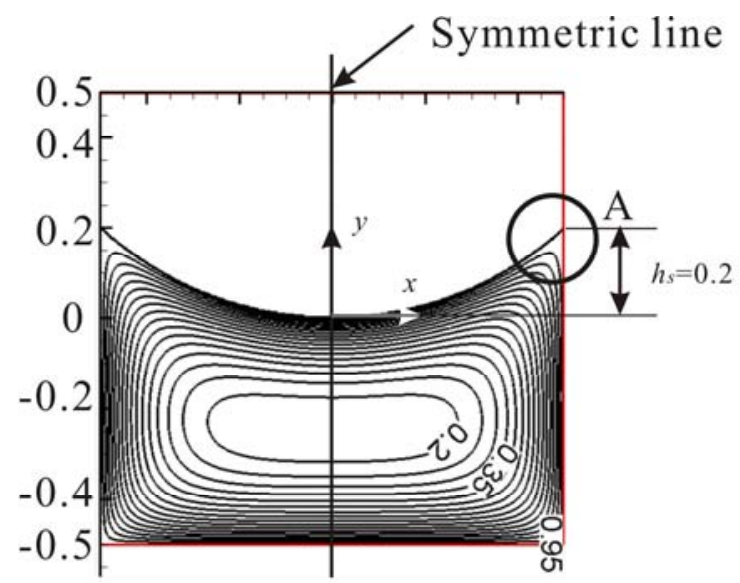

(a) Concave interface

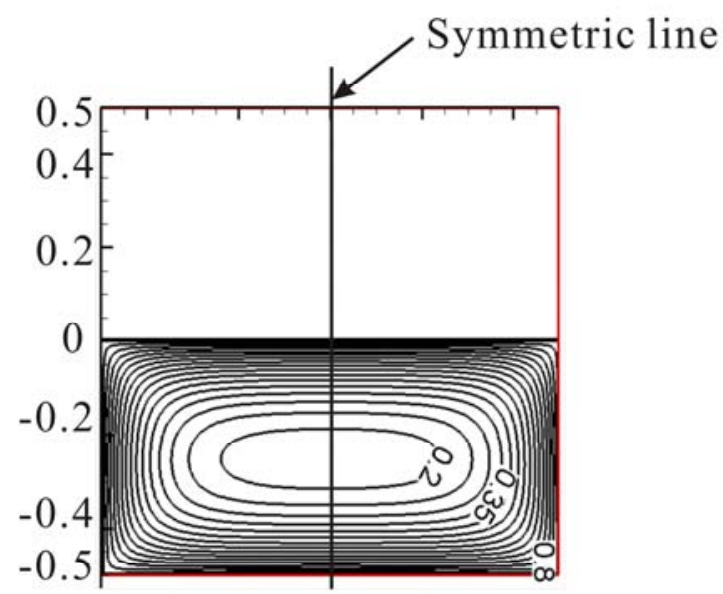

(b) plane interface

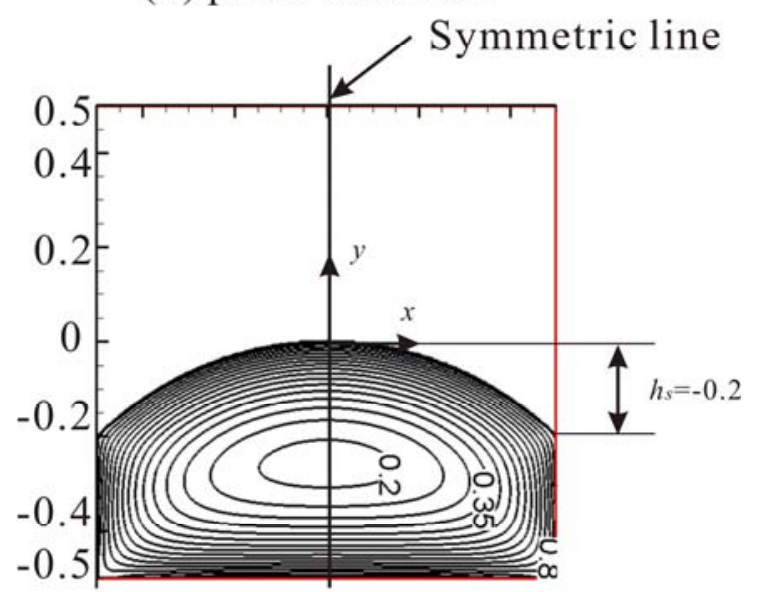

(c) convex interface

Fig. 6 The electric potential distributions of conducting fluid 1 


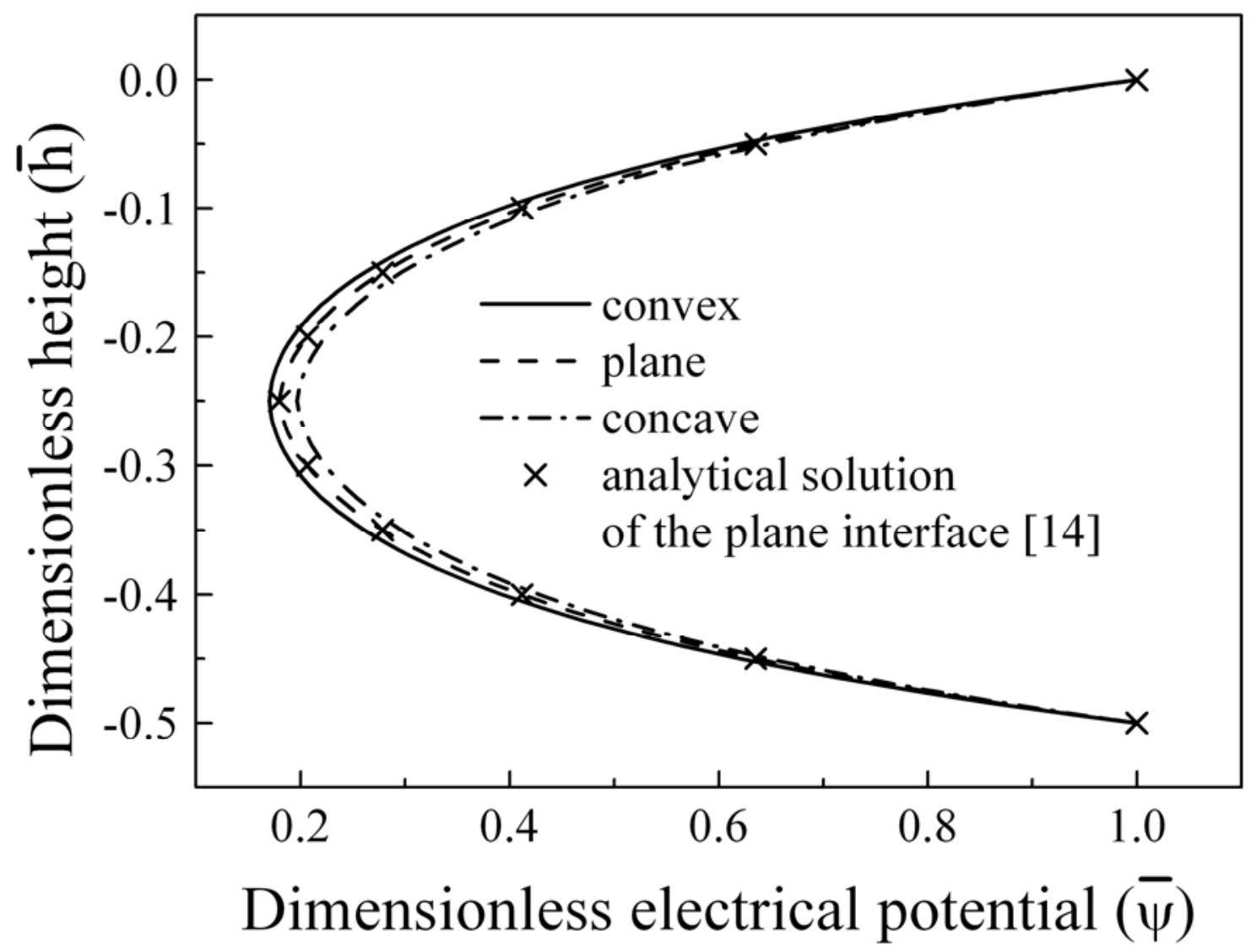

Fig. 7 Dimensionless electric potential distribution at the symmetric line in the conducting fluid 1 


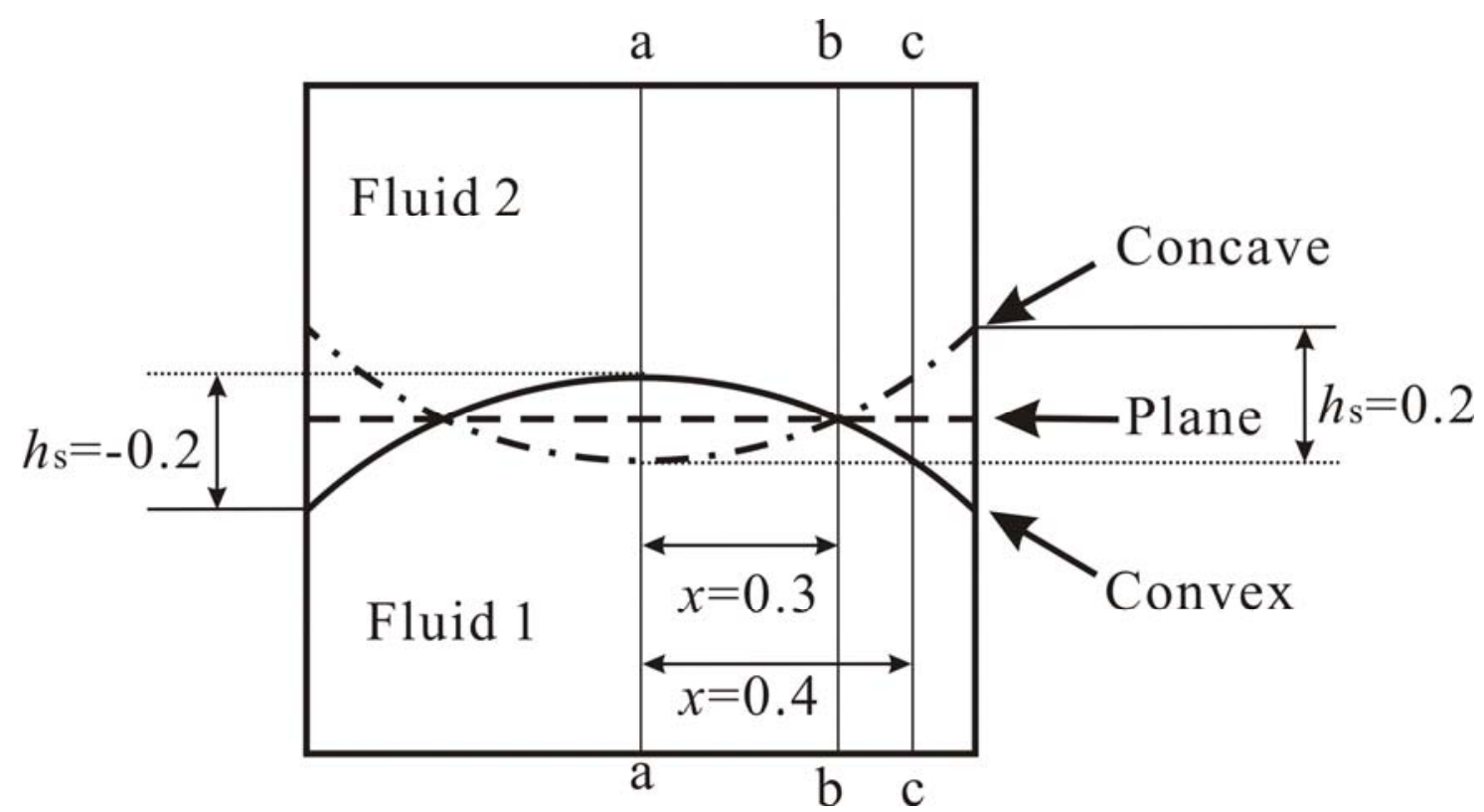

Fig. 8 Schematic illustration of the concave, plane and convex interface with the same liquid fraction of $0.5 e_{1}=e_{2}=0.5$ 


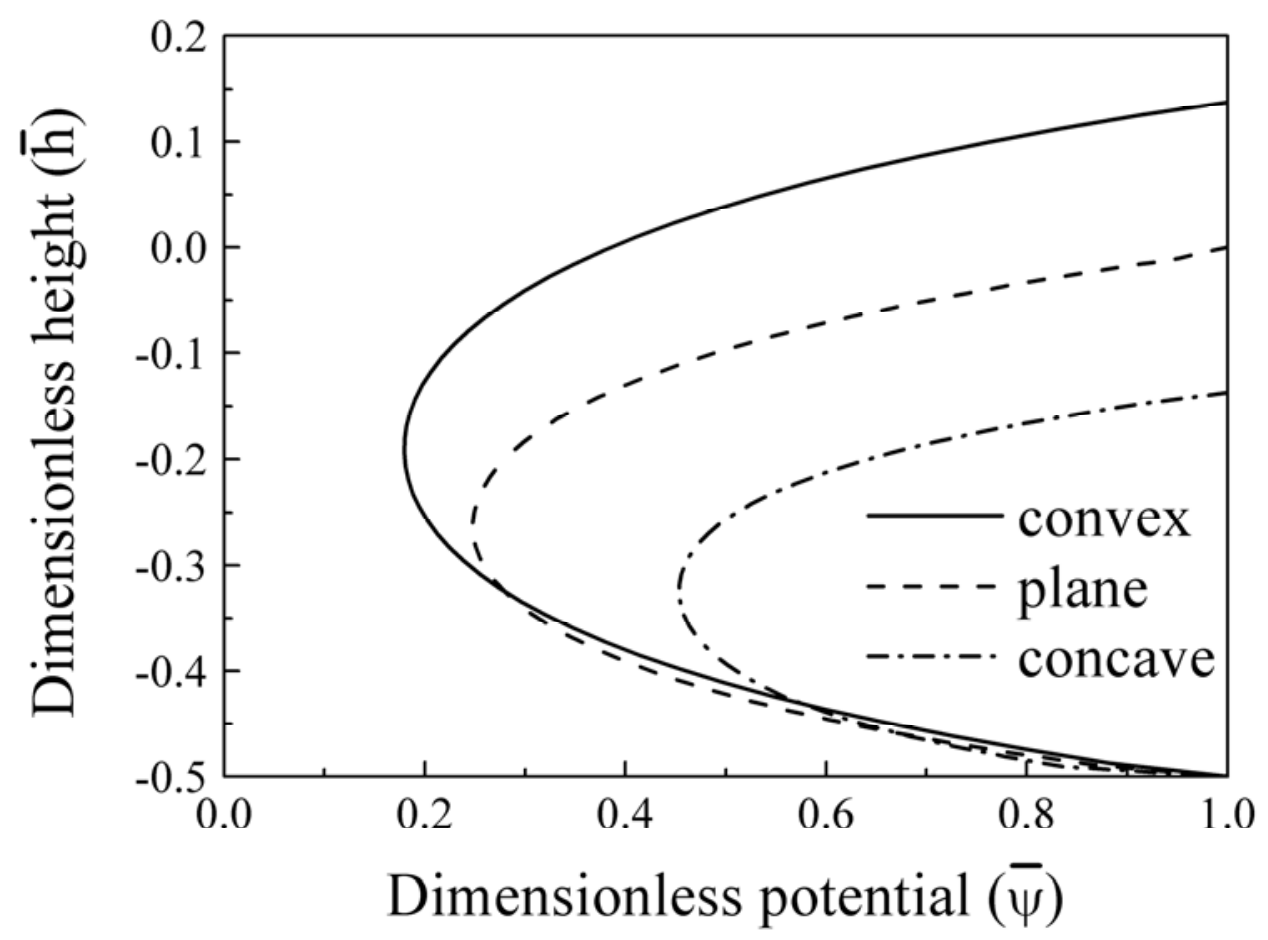

(a) Dimensionless electric potential distributions at section a-a

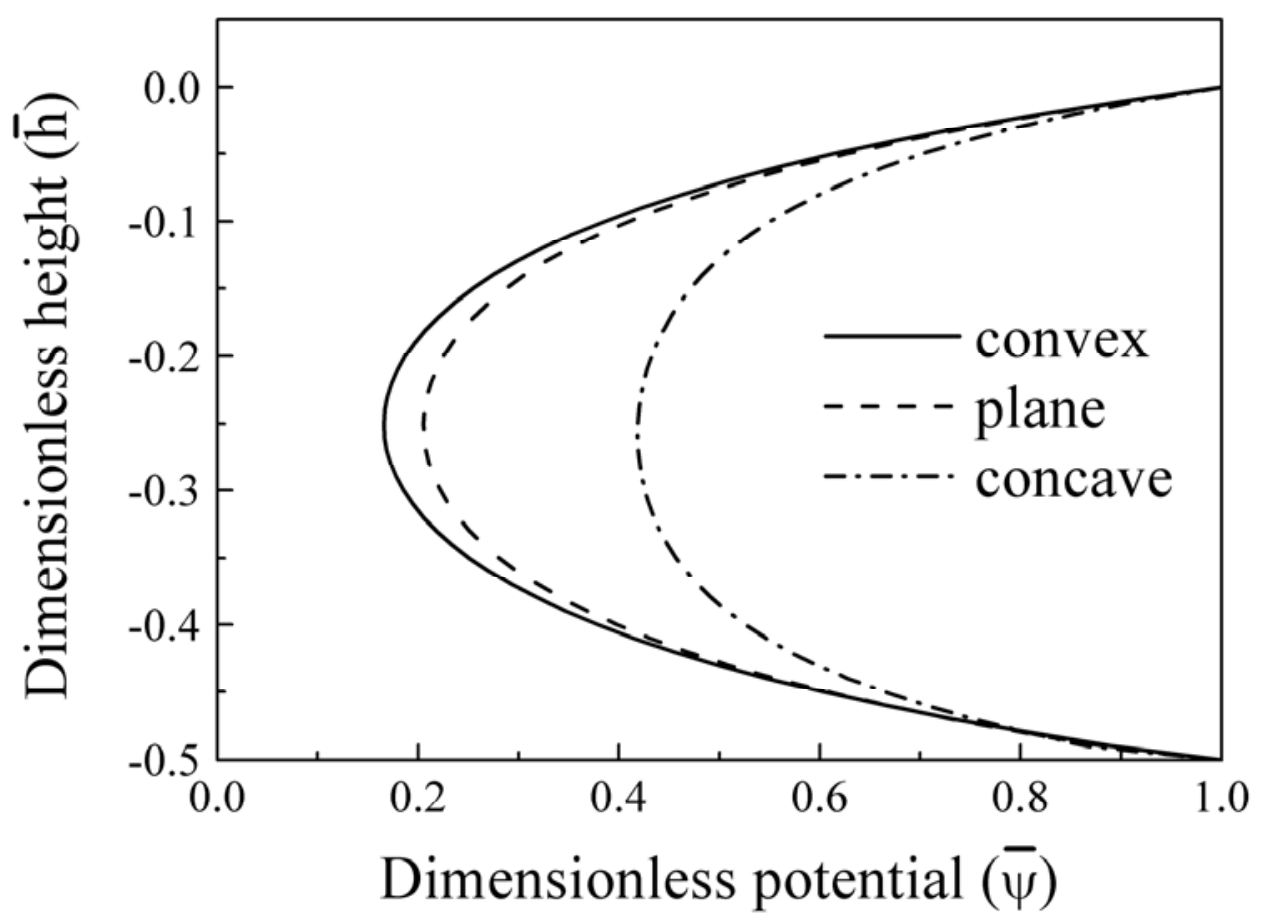

(b) Dimensionless electric potential distributions at section b-b 


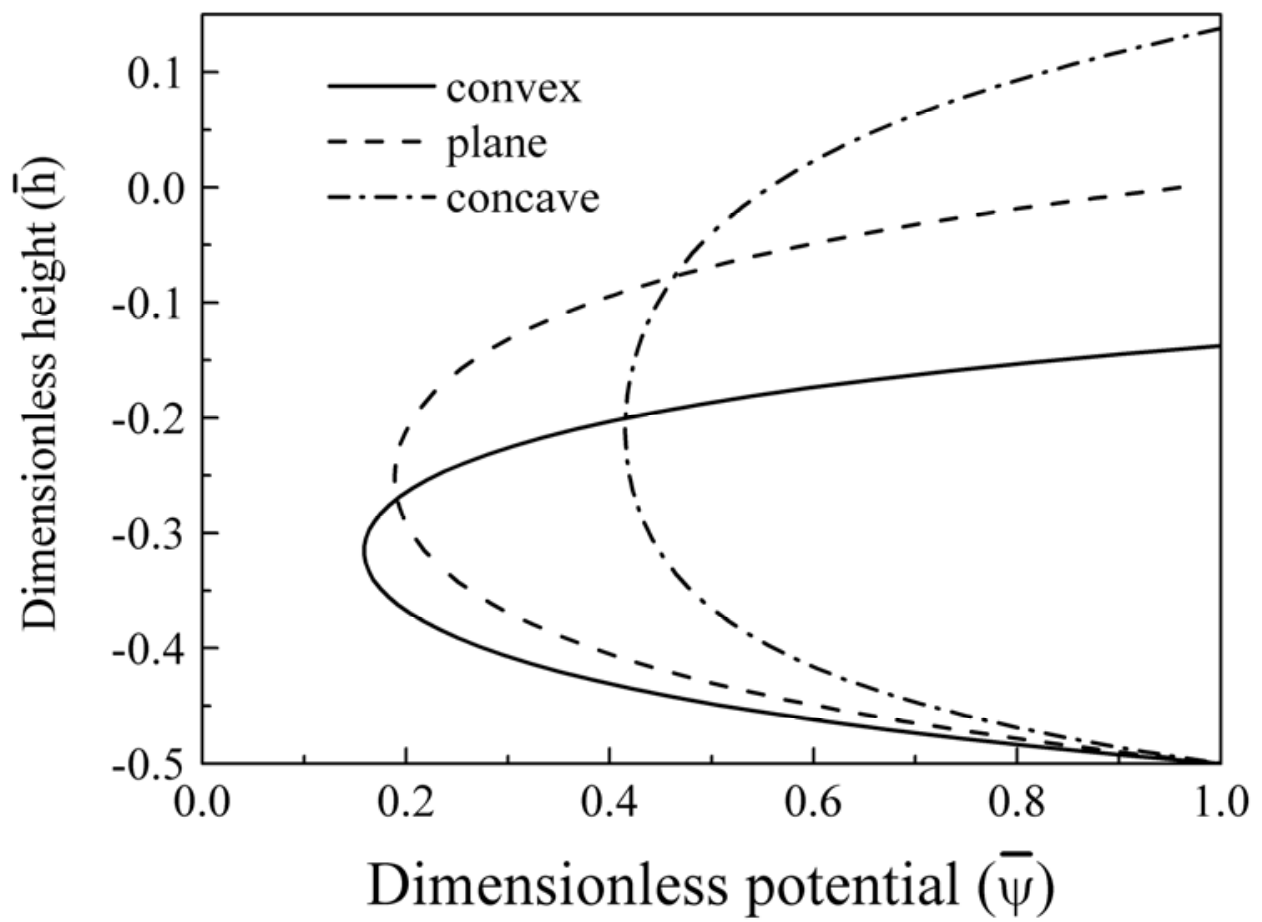

(c) Dimensionless electric potential distributions at section c-c

Fig. 9 Comparison of the dimensionless electric potential distribution at various sections

$$
\left(e_{1}=e_{2}=0.5, E_{\mathrm{x}}=1000, \bar{\mu}_{1}=\bar{\mu}_{2}=1\right)
$$




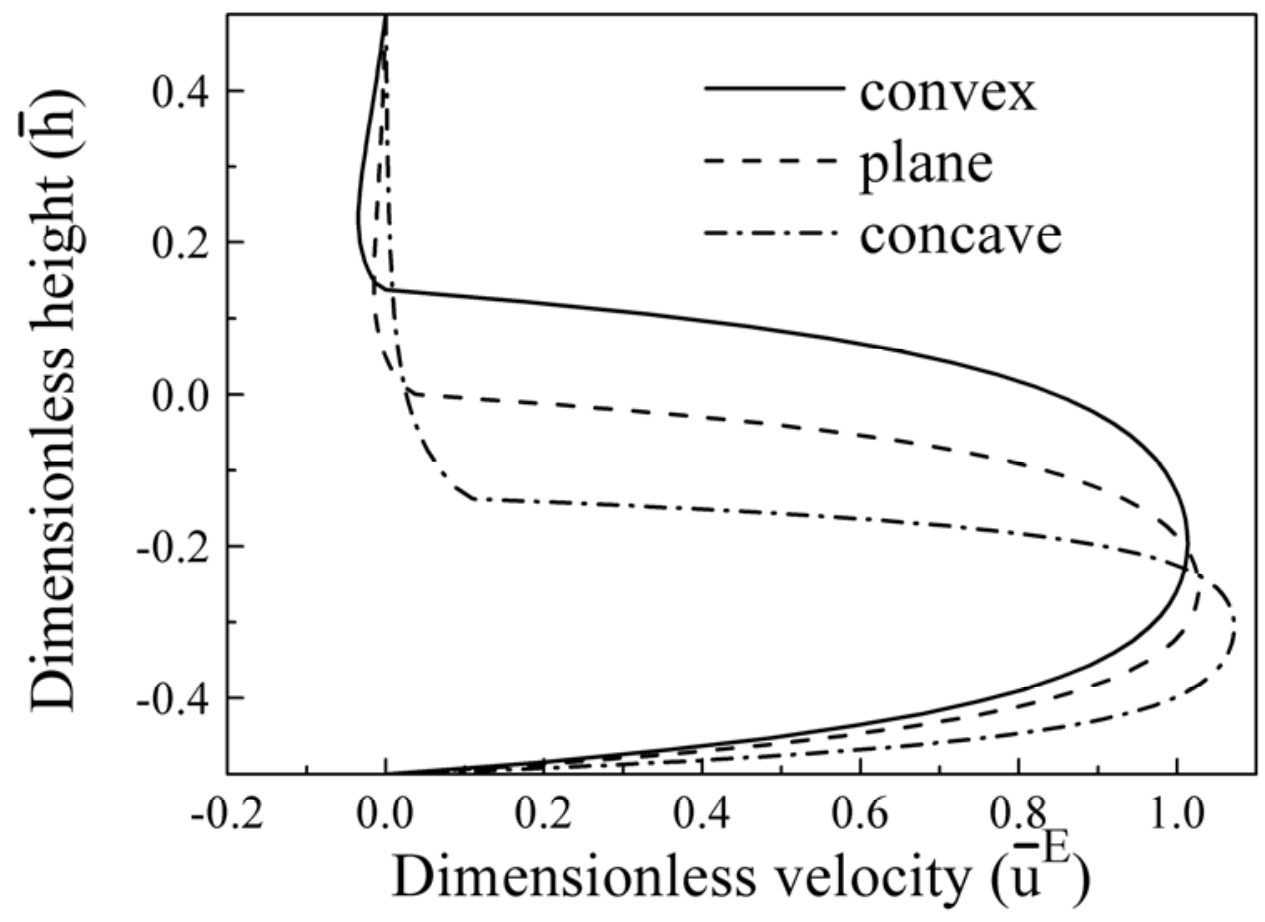

(a) Dimensionless electroosmotic velocity distribution at section a-a

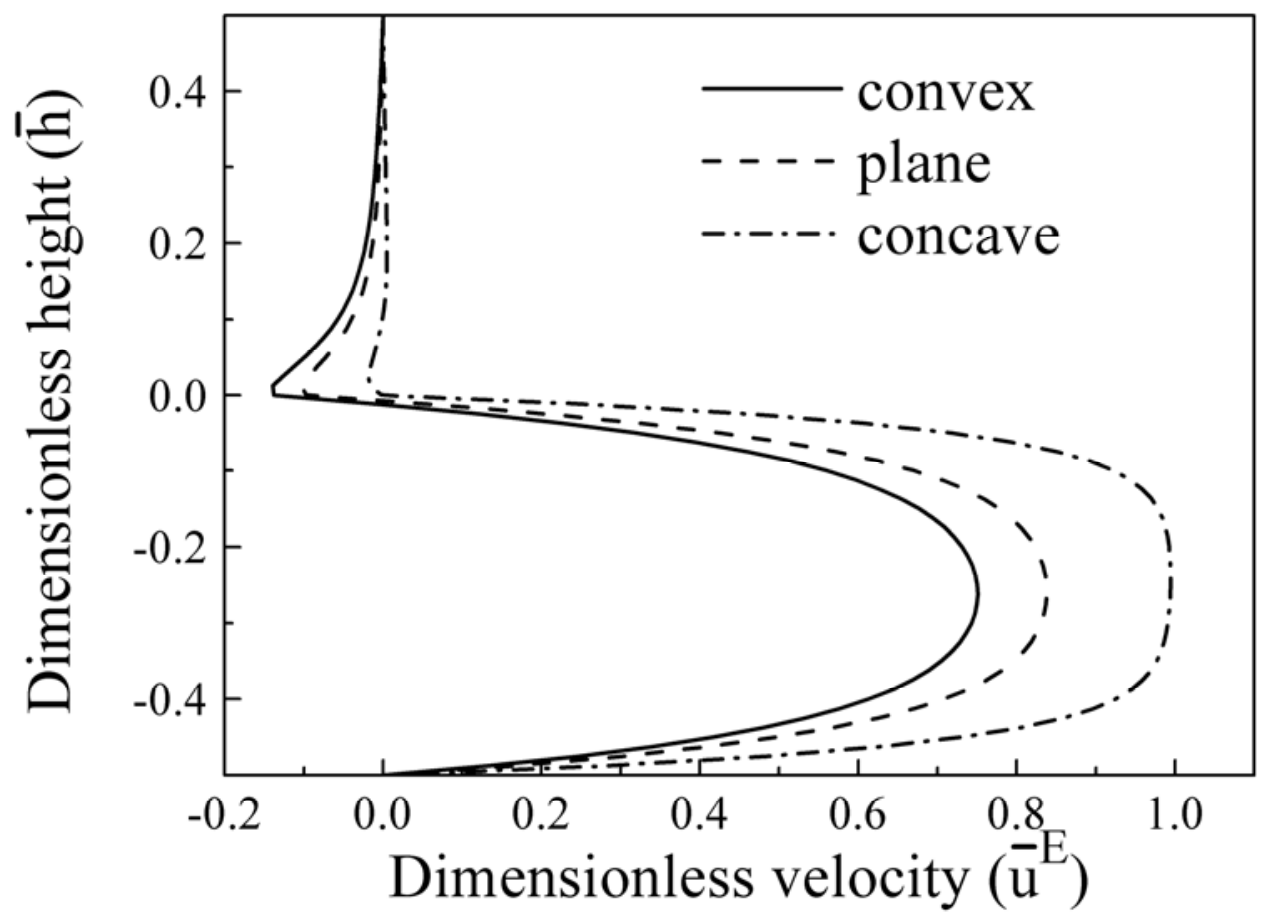

(b) Dimensionless electroosmotic velocity distribution at section b-b 


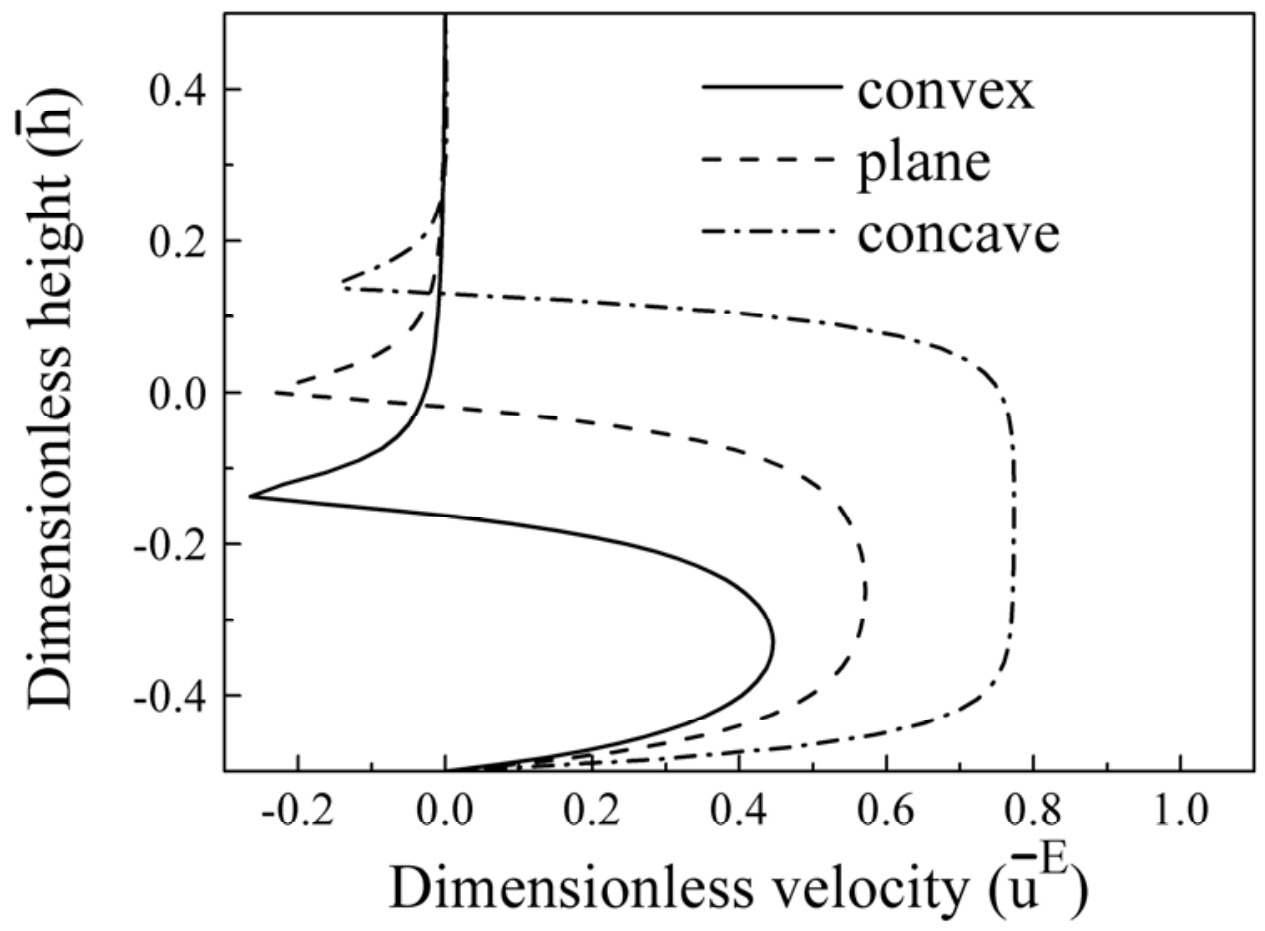

(c) Dimensionless electroosmotic velocity distribution at section c-c

Fig. 10 Dimensionless electroosmotic velocity distribution at various sections

$$
\left(e_{1}=e_{2}=0.5, E_{\mathrm{x}}=1000, d \bar{p} / d \bar{z}=0, \bar{\mu}_{1}=\bar{\mu}_{2}=1\right)
$$




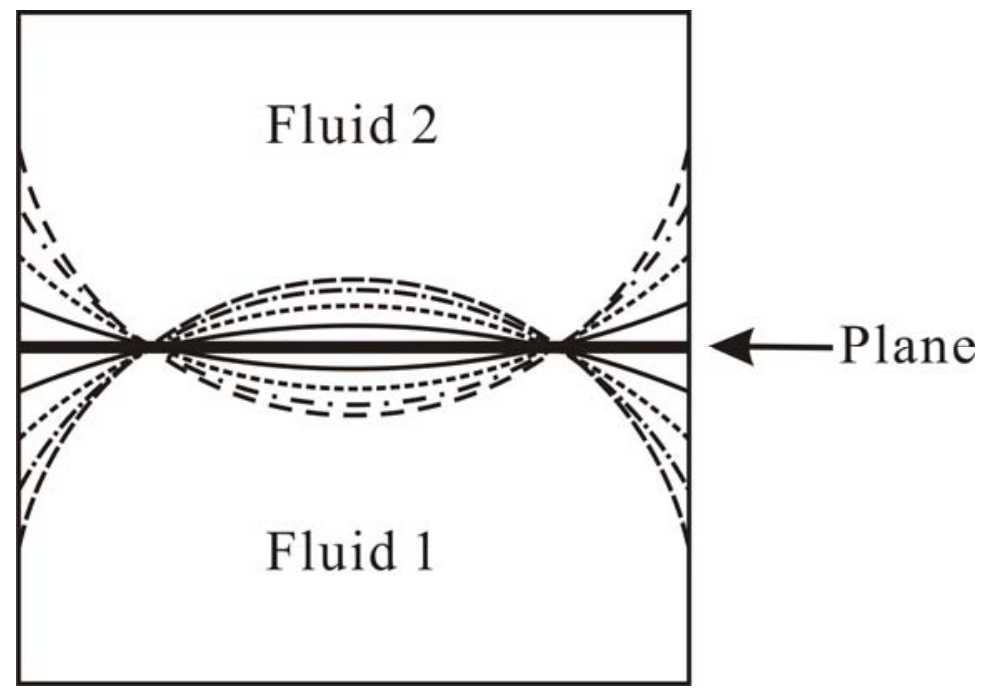

Fig. 11(a) Variation of the interface shapes with the contact angle $\left(e_{1}=e_{2}=0.5\right)$

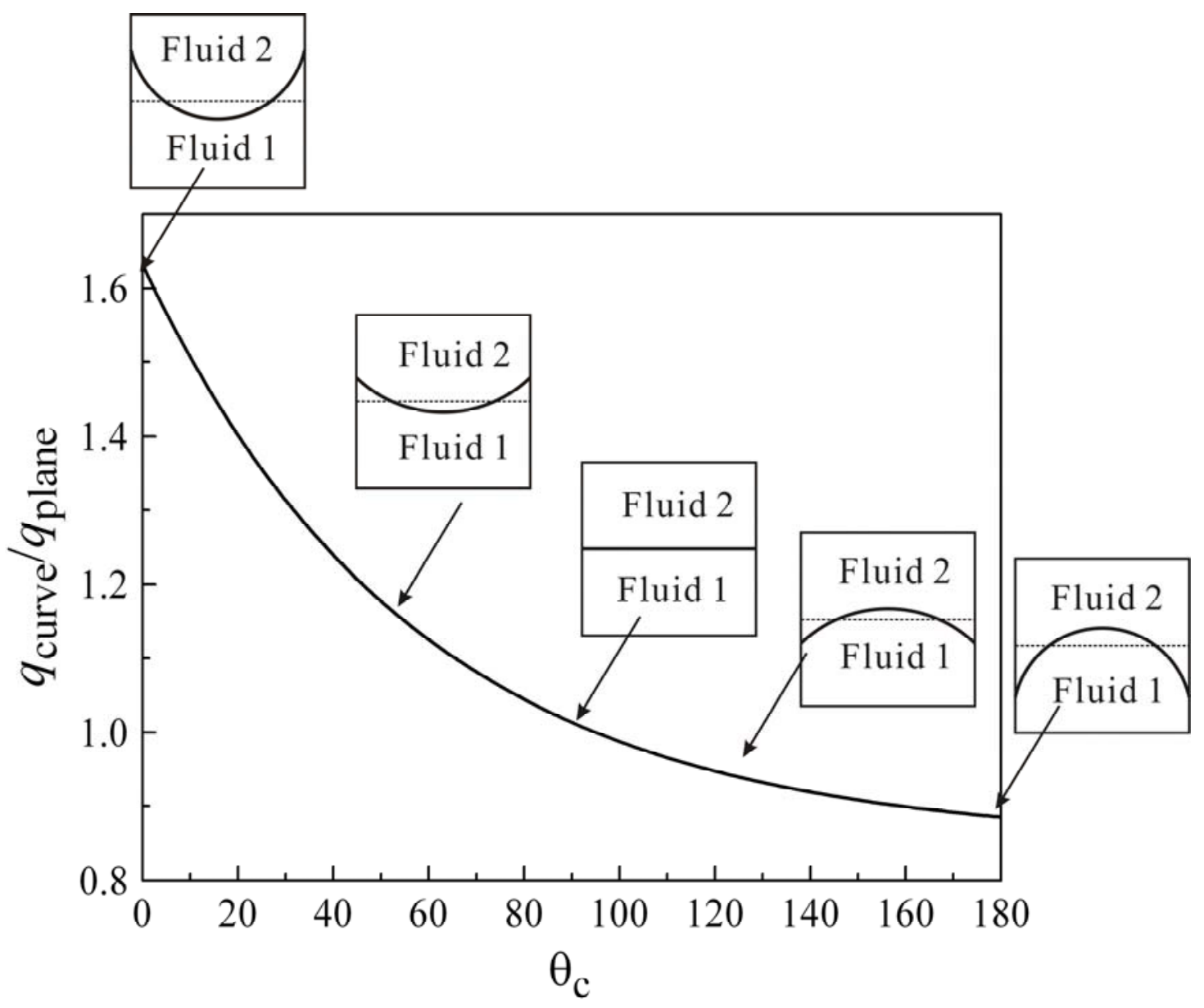

Fig. 11(b) Dimensionless volumetric flowrate of conducting fluid versus contact angle

$$
\left(e_{1}=e_{2}=0.5, E_{\mathrm{x}}=1000, \bar{\mu}_{1}=\bar{\mu}_{2}=1\right)
$$




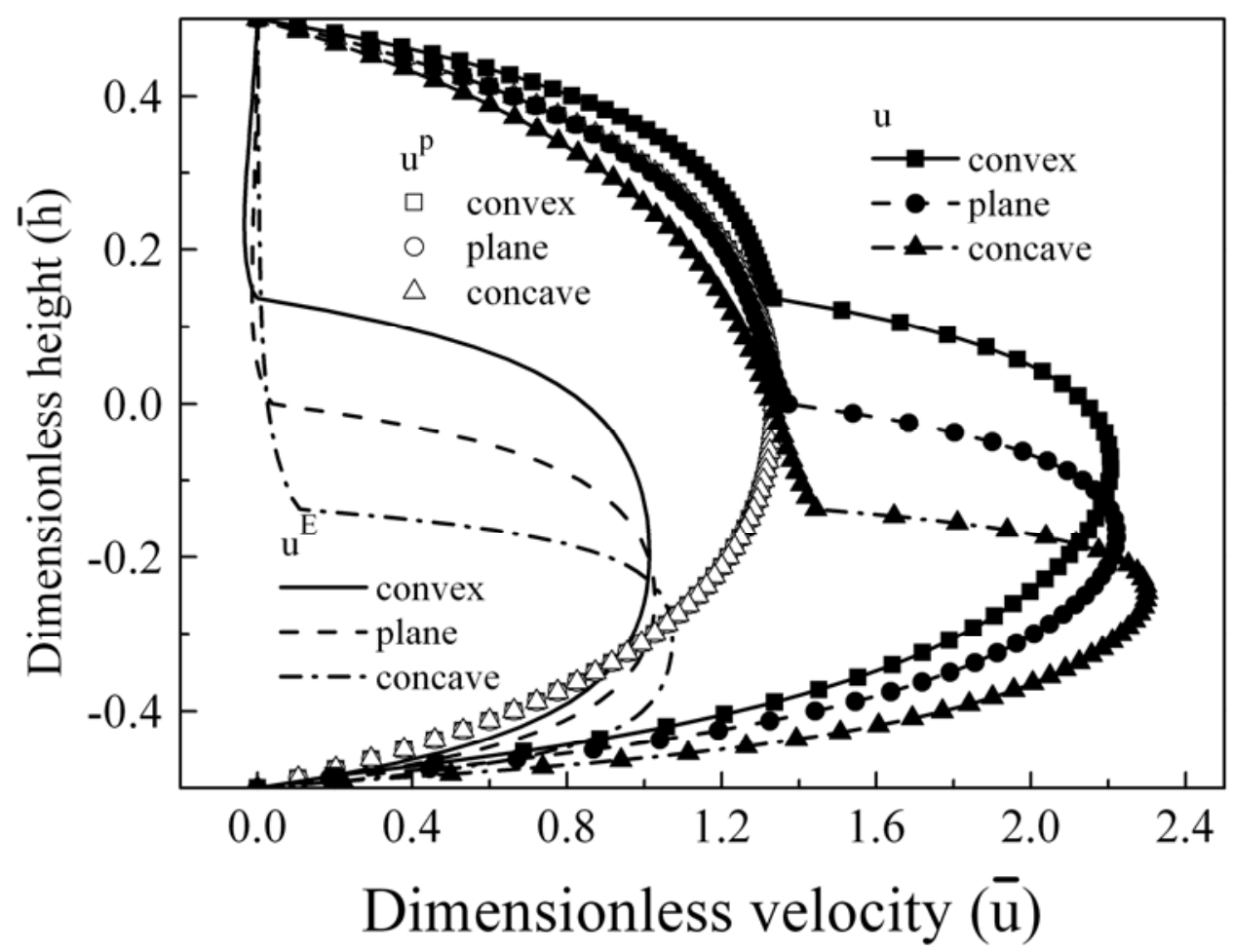

(a) Dimensionless velocity distribution at section a-a

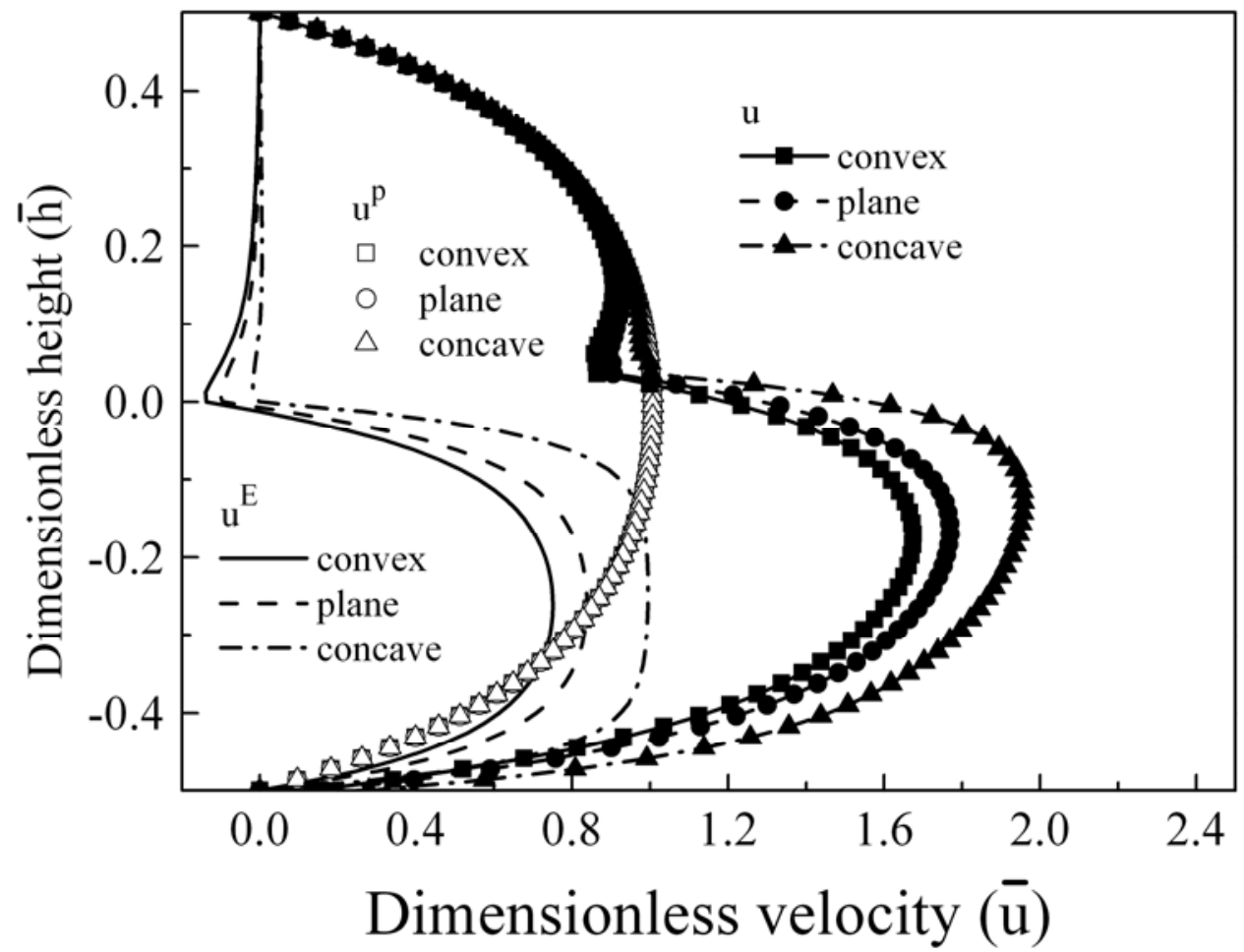

(b) Dimensionless velocity distribution at section b-b 


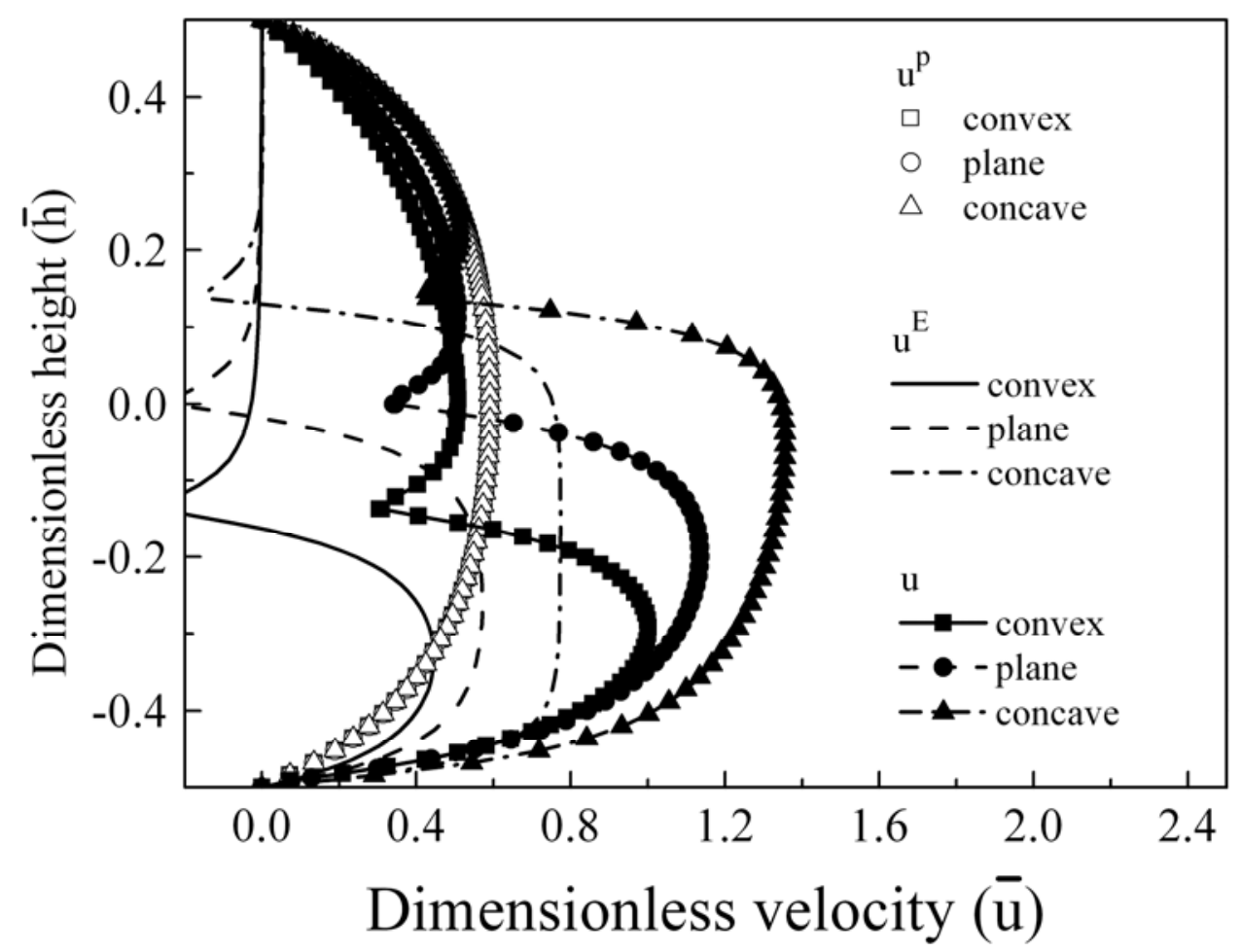

(c) Dimensionless velocity distribution at section c-c

Fig. 12 Dimensionless velocity distribution at various sections $\left(e_{1}=e_{2}=0.5, E_{\mathrm{x}}=1000, d \bar{p} / d \bar{z}=50000, \bar{\mu}_{1}=\bar{\mu}_{2}=1\right)$ 